\title{
On a Class of Integral Systems
}

\section{Volodymyr Derkach $^{1,2}$ (D) $\cdot$ Dmytro Strelnikov $^{1}$ (D) $\cdot$ Henrik Winkler $^{1}$}

Received: 23 March 2021 / Accepted: 21 July 2021

(c) The Author(s) 2021

\section{Abstract}

We study spectral problems for two-dimensional integral system with two given nondecreasing functions $R, W$ on an interval $[0, b)$ which is a generalization of the Krein string. Associated to this system are the maximal linear relation $T_{\max }$ and the minimal linear relation $T_{\min }$ in the space $L^{2}(d W)$ which are connected by $T_{\max }=T_{\min }^{*}$. It is shown that the limit point condition at $b$ for this system is equivalent to the strong limit point condition for the linear relation $T_{\max }$. In the limit circle case the Evans-Everitt condition is proved to hold on a subspace $T_{N}^{*}$ of $T_{\max }$ characterized by the Neumann boundary condition at $b$. The notion of the principal Titchmarsh-Weyl coefficient of this integral system is introduced. Boundary triple for the linear relation $T_{\max }$ in the limit point case (and for $T_{N}^{*}$ in the limit circle case) is constructed and it is shown that the corresponding Weyl function coincides with the principal Titchmarsh-Weyl coefficient of the integral system. The notion of the dual integral system is introduced by reversing the order of $R$ and $W$ and the formula relating the principal TitchmarshWeyl coefficients of the direct and the dual integral systems is proved. For every integral system with the principal Titchmarsh-Weyl coefficients $q$ a canonical system is constructed so that its Titchmarsh-Weyl coefficient $Q$ is the unwrapping transform of $q: Q(z)=z q\left(z^{2}\right)$.

Communicated by Harry Dym.

The research of the first author was supported by a grant of the German Research Foundation (DFG, Grant TR 903/22-1), by a grant of the Volkswagen Foundation and by Ministry of Education and Science of Ukraine (Project \# 0121U109525). The research of the second author was supported by Thuringian Graduate Scholarship programm.

$凶$ Volodymyr Derkach

volodymyr.derkach@tu-ilmenau.de

Dmytro Strelnikov

dmytro.strelnikov@tu-ilmenau.de

Henrik Winkler

henrik.winkler@tu-ilmenau.de

1 Institut für Mathematik, Technische Universität Ilmenau, Ilmenau, Germany

2 Department of Mathematics, Vasyl Stus Donetsk National University, Vinnytsya, Ukraine 
Keywords Integral systems · Krein strings - Dual systems · Principal

Titchmarsh-Weyl coefficient · Boundary triples · Canonical system

Mathematics Subject Classification Primary 34B24; Secondary 34L05 - 47A06 . 47A57 - 47B25 - 47E05

\section{Introduction}

In this paper spectral problems for integral systems, associated dual systems and, in particular, Krein strings are investigated. We consider an integral system of the form

$$
u(x, \lambda)=u(0, \lambda)-J \int_{0}^{x}\left[\begin{array}{cc}
\lambda d W(t) & 0 \\
0 & d R(t)
\end{array}\right] u(t, \lambda), \quad J=\left[\begin{array}{cc}
0 & -1 \\
1 & 0
\end{array}\right],
$$

where $u=\left[\begin{array}{ll}u_{1} & u_{2}\end{array}\right]^{T}$, with some spectral parameter $\lambda \in \mathbb{C}$ and measures $d W$ and $d R$ associated with non-decreasing functions $W(x)$ and $R(x)$ on an interval $[0, b)$, see [5].

Integral systems (1.1) arise in the theory of diffusion processes with two measures $[35,38]$. In the theory of stochastic processes the Eq. (1.3) describes generalized diffusion processes which includes both diffusion processes and birth and death processes $[18,19,23,31]$. The system (1.1) is reduced to a second order differential equation

$$
-\frac{d}{d W(x)}\left(\frac{d y}{d R(x)}\right)=\lambda y(x), \quad x \in[0, b), \quad \lambda \in \mathbb{C} \quad\left(y=u_{1}\right),
$$

with measure coefficients studied recently in [12] under an extra assumption that $R(x)$ is strictly monotone. If, in addition, $W(x)$ and $R(x)$ are absolutely continuous and $w:=W^{\prime}, p^{-1}:=R^{\prime}(>0$ a.e.) then the system (1.1) is reduced to the SturmLiouville equation in the polar form

$$
-\left(p y^{\prime}\right)^{\prime}=\lambda w y
$$

In a special case, when $R(x) \equiv x$ one has $u_{2}=u_{1}^{\prime}$ and system (1.1) can be rewritten as the equation of a vibrating string in the sense of Krein [27]

$$
y(x, \lambda)=y(0, \lambda)+x y^{\prime}(0, \lambda)-\lambda \int_{0}^{x}(x-t) y(t, s) d W(t), \quad x \in[0, b) .
$$

Let $c(\cdot, \lambda)$ and $s(\cdot, \lambda)$ be the unique solutions of (1.3) satisfying the initial conditions

$$
c(0, \lambda)=1, c^{\prime}(0, \lambda)=0, \quad \text { and } s(0, \lambda)=0, s^{\prime}(0, \lambda)=1 .
$$

The function

$$
q_{S}(\lambda):=\lim _{x \rightarrow b} \frac{s(x, \lambda)}{c(x, \lambda)}
$$


is called the principal Titchmarsh-Weyl coefficient of the string [30] or the dynamic compliance coefficient in the terminology of Kac and Krein [27] and describes the spectral properties of the string. The principal Titchmarsh-Weyl coefficient $q(\lambda)$ is a Stieltjes function and the measure $d \sigma$ from its integral representation

$$
q_{S}(\lambda)=a+\int_{0}^{\infty} \frac{d \sigma(t)}{t-\lambda}, \quad a \geq 0
$$

is the spectral measure of the string (1.3), which in the limit point case at $b$ is specified by the boundary condition $u^{\prime}(0)=0$ at 0 .

Denote the integral system (1.1) by $S[R, W]$. In the present paper we define the principal Titchmarsh-Weyl coefficient $q$ of the integral system $S[R, W]$ by

$$
q(\lambda):=\lim _{x \rightarrow b} \frac{s_{1}(x, \lambda)}{c_{1}(x, \lambda)},
$$

where $\left[c_{1}(\cdot, \lambda) c_{2}(\cdot, \lambda)\right]^{T}$, and $\left[s_{1}(\cdot, \lambda) s_{2}(\cdot, \lambda)\right]^{T}$ are solutions of (1.1) satisfying the initial conditions

$$
c_{1}(0, \lambda)=1, c_{2}(0, \lambda)=0, \quad \text { and } s_{1}(0, \lambda)=0, s_{2}(0, \lambda)=1 \text {. }
$$

Formula (1.6) requires justification. For this purpose we use the operator approach to the integral system $S[R, W]$ developed in [41], the boundary triples technique from [21,32] and the theory of associated Weyl functions as introduced in $[10,11]$. The maximal linear relation $T_{\max }$ is defined (see Definition 2.7) as the set of pairs $\boldsymbol{u}=\left[\begin{array}{ll}u_{1} & f\end{array}\right]^{T}$ such that $u_{1}, f \in L^{2}(d W)$ and the equation (2.17) is satisfied for some function $u_{2} \in B V_{\mathrm{loc}}[0, b)$, i.e. of bounded variation on $\left[0, b^{\prime}\right)$ for every $b^{\prime}<b$. The closure of its restriction to the set of compactly supported functions is called the minimal linear relation $T_{\min }$. In [41] it was shown that $T_{\min }$ is symmetric in $L^{2}(d W)$, $T_{\max }=T_{\min }^{*}$ and boundary triples for the linear relation $T_{\min }$ were constructed both in the limit point and in the limit circle case.

In Theorem 4.3 we show that the system $S[R, W]$ is in the limit point case at $b$ if and only if it satisfies the strong limit point condition at $b$, see [16], which in our case is of the form

$$
\lim _{x \rightarrow b} u_{1}(x) u_{2}(x)=0 \text { for all } \boldsymbol{u} \in T_{\max } .
$$

As a consequence of (1.8) we conclude that in the limit point case the linear relation $T_{\min }$ and its von Neumann extension $A_{N}$, characterized by the boundary condition $u_{2}(0)=0$, are nonnegative, the corresponding Weyl function is a Stieltjes function and coincides with the principal Titchmarsh-Weyl coefficient of the system $S[R, W]$. The strong limit point condition for second order differential operators was introduced by Everitt [16].

In the limit circle case the linear relation $T_{\min }$ has defect numbers $(2,2)$, in this case an intermediate symmetric extension $T_{N}$ with defect numbers $(1,1)$ of $T_{\min }$ is considered as the restriction of $T_{\max }$ to the set of elements $\boldsymbol{u} \in T_{\max }$ such that $u_{1}(0)=u_{2}(0)=u_{2}(b)=0$. In this case we show in Lemma 3.3 that the strong 
limit point condition (1.8) fails to hold, but still the limit in (1.8) is vanishing on the subspace $T_{N}^{*}$ of $T_{\max }$, i.e. the following Evans-Everitt condition holds, cf. [17]:

$$
\lim _{x \rightarrow b} u_{1}(x) u_{2}(x)=0 \text { for all } \boldsymbol{u} \in T_{N}^{*}
$$

This result implies the nonnegativity of the linear relation $T_{N}$.

In [33] another analytical object—the Neumann m-function of the system $S[R, W]$ was introduced by the equality

$$
m_{N}(\lambda):=\lim _{x \rightarrow b} \frac{s_{2}(x, \lambda)}{c_{2}(x, \lambda)}
$$

which is a special case of a more general definition of the Neumann $m$-function presented in [5]. In Proposition 3.6 it is shown that the Neumann $m$-function $m_{N}(\lambda)$ is a Stieltjes function and it coincides with the principal Titchmarsh-Weyl coefficient of the integral system $S[R, W]$.

The system $S[R, W]$ is called regular if $R(b)+W(b)<\infty$ and singular otherwise. In the regular case we construct the canonical singular extension $S[\widetilde{R}, \widetilde{W}]$ of the system $S[R, W]$ with $R, W$ extended to non-decreasing functions $\widetilde{R}, \widetilde{W}$ on the interval $(0, \infty)$, so that the principal Titchmarsh-Weyl coefficients of both systems coincide.

The dual system $\widehat{S}[R, W]$ of the integral system $S[R, W]$ in the singular case is obtained by changing the roles of $R$ and $W$. In the regular case the dual system of the integral system $S[R, W]$ is defined as the dual of the canonical singular extension $S[\widetilde{R}, \widetilde{W}]$ of the system $S[R, W]$. In Theorem 5.2 it is shown that the principal Titchmarsh-Weyl coefficient $\widehat{q}$ of the dual system is related to the principal Titchmarsh-Weyl coefficient $q$ of the system $S[R, W]$ by the equality

$$
\widehat{q}(\lambda)=-\frac{1}{\lambda q(\lambda)}
$$

both in the regular and the singular case.

In Theorem 6.1 given a singular integral system $S(R, W)$ we construct a canonical system

$$
J y^{\prime}(x)=-z H_{d}(x) y(x), x \in\left[0, l_{H}\right), \quad y_{1}(0)=0,
$$

with a diagonal Hamiltonian

$$
H_{d}(x)=\left[\begin{array}{cc}
h_{1}(x) & 0 \\
0 & h_{2}(x)
\end{array}\right]
$$

such that the corresponding Titchmarsh-Weyl coefficient $Q_{d}$ (see [7]) is connected with the principal Titchmarsh-Weyl coefficient $q$ of the integral system $S(R, W)$ by the formula

$$
Q_{d}(z)=z q\left(z^{2}\right) .
$$

In the case of a string $(R(x) \equiv x)$ the notion of the dual string and formula (1.11) connecting the principal Titchmarsh-Weyl coefficients of the direct and the dual string 
in the singular case were presented in [25,27]. Analogues of the relations (1.11) and (1.13) between strings, dual strings and canonical systems of differential equations were studied also in [30].

\section{Preliminaries}

\subsection{Linear Relations}

Let $\mathfrak{H}$ be a Hilbert space. A linear relation $T$ in $\mathfrak{H}$ is a linear subspace of $\mathfrak{H} \times \mathfrak{H}$. Let us recall some basic definitions and properties associated with linear relations, see $[1,4]$.

The domain, the range, the kernel, and the multivalued part of a linear relation $T$ are defined as follows:

$$
\begin{aligned}
\operatorname{dom} T:=\left\{f:\left[\begin{array}{l}
f \\
g
\end{array}\right] \in T\right\}, & \operatorname{ran} T:=\left\{g:\left[\begin{array}{l}
f \\
g
\end{array}\right] \in T\right\}, \\
\operatorname{ker} T:=\left\{f:\left[\begin{array}{l}
f \\
0
\end{array}\right] \in T\right\}, & \operatorname{mul} T:=\left\{g:\left[\begin{array}{l}
0 \\
g
\end{array}\right] \in T\right\} .
\end{aligned}
$$

The adjoint linear relation $T^{*}$ is defined by

$$
T^{*}:=\left\{\left[\begin{array}{l}
u \\
f
\end{array}\right] \in \mathfrak{H} \times \mathfrak{H}:\langle f, v\rangle_{\mathfrak{H}}=\langle u, g\rangle_{\mathfrak{H}} \text { for any }\left[\begin{array}{l}
v \\
g
\end{array}\right] \in T\right\}
$$

A linear relation $T$ in $\mathfrak{H}$ is called closed if $T$ is closed as a subspace of $\mathfrak{H} \times \mathfrak{H}$. The set of all closed linear operators (relations) is denoted by $\mathcal{C}(\mathfrak{H})(\widetilde{\mathcal{C}}(\mathfrak{H}))$. Identifying a linear operator $T \in \mathcal{C}(\mathfrak{H})$ with its graph one can consider $\mathcal{C}(\mathfrak{H})$ as a part of $\widetilde{\mathcal{C}}(\mathfrak{H})$.

Let $T$ be a closed linear relation, $\lambda \in \mathbb{C}$, then

$$
T-\lambda I:=\left\{\left[\begin{array}{c}
f \\
g-\lambda f
\end{array}\right]:\left[\begin{array}{l}
f \\
g
\end{array}\right] \in T\right\}
$$

A point $\lambda \in \mathbb{C}$ such that $\operatorname{ker}(T-\lambda I)=\{0\}$ and $\operatorname{ran}(T-\lambda I)=\mathfrak{H}$ is called a regular point of the linear relation $T$. Let $\rho(T)$ be the set of regular points. The point spectrum $\sigma_{p}(T)$ of the linear relation $T$ is defined by

$$
\sigma_{p}(T):=\{\lambda \in \mathbb{C}: \operatorname{ker}(T-\lambda I) \neq\{0\}\}
$$

A linear relation $T$ is called symmetric if $T \subseteq T^{*}$. A point $\lambda \in \mathbb{C}$ is called a point of regular type (and is written as $\lambda \in \widehat{\rho}(T)$ ) for a closed symmetric linear relation $T$, if $\lambda \notin \sigma_{p}(T)$ and the subspace $\operatorname{ran}(T-\lambda I)$ is closed in $H$. For $\lambda \in \widehat{\rho}(T)$ let us set $\mathfrak{N}_{\lambda}\left(T^{*}\right):=\operatorname{ker}\left(T^{*}-\lambda I\right)$ and

$$
\widehat{\mathfrak{N}}_{\lambda}\left(T^{*}\right):=\left\{\boldsymbol{u}_{\lambda}=\left[\begin{array}{c}
u_{\lambda} \\
\lambda u_{\lambda}
\end{array}\right]: u_{\lambda} \in \mathfrak{N}_{\lambda}\left(T^{*}\right)\right\} .
$$


The deficiency indices of a symmetric linear relation $T$ are defined as

$$
n_{ \pm}(T):=\operatorname{dim} \operatorname{ker}\left(T^{*} \mp i I\right) .
$$

\subsection{Boundary Triples and Weyl Functions}

Let $T$ be a symmetric linear relation with deficiency indices $(1,1)$. In the case of a densely defined operator the notion of the boundary triple was introduced in [21,32]. Following the papers $[11,37]$ we shall give a definition of a boundary triple for the linear relation $T^{*}$.

Definition 2.1 A tuple $\Pi=\left(\mathbb{C}, \Gamma_{0}, \Gamma_{1}\right)$, where $\Gamma_{0}$ and $\Gamma_{1}$ are linear mappings from $T^{*}$ to $\mathbb{C}$, is called a boundary triple for the linear relation $T^{*}$, if:

(i) for all $\boldsymbol{u}=\left[\begin{array}{l}u \\ f\end{array}\right], \boldsymbol{v}=\left[\begin{array}{l}v \\ g\end{array}\right] \in T^{*}$ the following generalized Green's identity holds

$$
\langle f, v\rangle_{\mathfrak{H}}-\langle u, g\rangle_{\mathfrak{H}}=\Gamma_{1} u \overline{\Gamma_{0} v}-\Gamma_{0} u \overline{\Gamma_{1} \boldsymbol{v}}
$$

(ii) the mapping $\Gamma=\left[\begin{array}{l}\Gamma_{0} \\ \Gamma_{1}\end{array}\right]: T^{*} \rightarrow \mathbb{C}^{2}$ is surjective.

Notice, that in contrast to [37] the linear relation $T$ is not supposed to be singlevalued. The following linear relations

$$
A_{0}:=\operatorname{ker} \Gamma_{0}, \quad A_{1}:=\operatorname{ker} \Gamma_{1}
$$

are selfadjoint extensions of the symmetric linear relation $T$.

Definition $2.2([10,11])$ Let $\Pi=\left(\mathbb{C}, \Gamma_{0}, \Gamma_{1}\right)$ be a boundary triple for the linear relation $T^{*}$. The scalar function $m(\cdot)$ and the vector valued function $\gamma(\cdot)$ defined by

$$
m(\lambda) \Gamma_{0} \boldsymbol{u}_{\lambda}=\Gamma_{1} \boldsymbol{u}_{\lambda}, \quad \gamma(\lambda) \Gamma_{0} \boldsymbol{u}_{\lambda}=u_{\lambda}, \quad \boldsymbol{u}_{\lambda}=\left[\begin{array}{c}
u_{\lambda} \\
\lambda u_{\lambda}
\end{array}\right] \in \widehat{\mathfrak{N}}_{\lambda}\left(T^{*}\right), \quad \lambda \in \rho\left(A_{0}\right)
$$

are called the Weyl function and the $\gamma$-field of the symmetric linear relation $T$ corresponding to the boundary triple $\Pi$.

The Weyl function and the $\gamma$-field are connected via the next identity (see [11])

$$
m(\lambda)-m(\zeta)^{*}=(\lambda-\bar{\zeta}) \gamma(\zeta)^{*} \gamma(\lambda), \quad \lambda, \zeta \in \rho\left(A_{0}\right)
$$

Definition 2.3 ([26]) A function $m: \mathbb{C} \backslash \mathbb{R} \rightarrow \mathcal{B}(\mathcal{H})$ is said to be a Herglotz-Nevanlinna function and is written as $m \in \mathcal{N}$, if the following conditions hold:

(i) $m$ is holomorphic in $\mathbb{C} \backslash \mathbb{R}$;

(ii) $\operatorname{Im} m(\lambda) \geq 0$ for $\lambda \in \mathbb{C}_{+}:=\{\lambda \in \mathbb{C}: \operatorname{Im} \lambda>0\}$;

(iii) $m(\bar{\lambda})=m(\lambda)^{*}$ for $\lambda \in \mathbb{C} \backslash \mathbb{R}$. 
It follows from (2.11) that the Weyl function $m(\cdot)$ is a Herglotz-Nevanlinna function. A Herglotz-Nevanlinna function $m$ which admits a holomorphic continuation to $\mathbb{R}_{-}$ and takes nonnegative values for all $\lambda \in \mathbb{R}_{-}$is called a Stieltjes function. Every Stieltjes function $m$ admits an integral representation (1.5) with a non-decreasing function $\sigma(t)$ such that $\int_{\mathbb{R}_{+}}(1+t)^{-1} d \sigma(t)<\infty$.

\subsection{Minimal and Maximal Relations Associated with the Integral System $S[R, W]$}

Let $I=[0, b)$ be an interval with $b \leq \infty$, let $W(x)$ be a non-decreasing left-continuous function on $I$ such that $W(0)=0$, let $d W$ be the corresponding Lebesgue-Stieltjes measure, and let $\mathcal{L}^{2}(d W, I)$ be an inner product space which consists of complex valued functions $f$ such that

$$
\int_{I}|f(t)|^{2} d W(t)<\infty
$$

with inner product defined by

$$
\langle f, g\rangle_{W}=\int_{I} f(t) \overline{g(t)} d W(t) .
$$

$\mathcal{L}_{\text {comp }}^{2}(d W, I)$ denotes the subspace consisting of those $f \in \mathcal{L}^{2}(d W, I)$ with compact support in $I, B V[0, b)$ denotes the set of functions of bounded variation on $[0, b)$ and $B V_{\text {loc }}[0, b)$ is the set of functions $f$ such that $f \in B V\left[0, b^{\prime}\right)$ for every $b^{\prime}<b$. Denote by $L^{2}(d W, I)$ the corresponding quotient space for $\mathcal{L}^{2}(d W, I)$, which consists of equivalence classes w.r.t. $d W$ and denote by $\pi$ the corresponding quotient map, i.e. $\pi: \mathcal{L}^{2}(d W, I) \rightarrow L^{2}(d W, I)$. Often we write $L^{2}(d W)$ instead of $L^{2}(d W, I)$ if $I$ coincides with $[0, b)$.

From now on the following convention is used for the integration limits for any measure $d W$ on an interval:

$$
\int_{a}^{x} f d W:=\int_{[a, x)} f d W .
$$

Thus, an integral as a function of its upper limit is always left-continuous. With every function of bounded variation $f$ we associate the left-continuous and the rightcontinuous functions $f_{-}$and $f_{+}$defined by

$$
f_{-}(x):=\lim _{t \uparrow x} f(t), \quad f_{+}(x):=\lim _{t \downarrow x} f(t) .
$$

Let $u$ and $v$ be left-continuous functions of bounded variation, $d u$ and $d v$ be the corresponding Lebesgue-Stieltjes measures. The following integration-by-parts formula for the Lebesgue-Stieltjes integral (see e.g. [22]) is used throughout the paper

$$
\int_{a}^{x} u d v+\int_{a}^{x} v_{+} d u=u(x) v(x)-u(a) v(a) .
$$


If $u$ and $u_{+}$have no zeros then it follows with $v=1 / u$ from (2.14)

$$
d(1)=d\left(\frac{u}{u}\right)=u d\left(\frac{1}{u}\right)+\frac{1}{u_{+}} d u=0 .
$$

This leads to the quotient-rule formula

$$
d\left(\frac{1}{u}\right)=-\frac{d u}{u u_{+}} .
$$

The following existence and uniqueness theorem for integral systems was proved in [5, Theorem 1.1].

Theorem 2.4 Let $d S$ be a complex $n \times n$ matrix-valued measure. For every left continuous (either $n \times n$ or $n \times 1$ matrix valued) function $A(x)$ in $B V_{\mathrm{loc}}[0, b)$ there is a unique function $U$ such that the equality

$$
U(x)=A(x)+\int_{0}^{x} d S \cdot U
$$

holds for every point $x \in[0, b)$.

Remark 2.5 Due to the properties of the Lebesgue-Stieltjes integral and the used convention, any solution $U$ to (2.16) is left continuous and belongs to $B V_{\text {loc }}[0, b)$, componentwise.

Now we focus on integral systems $S[R, W]$ of the form (1.1), where $R(x)$ and $W(x)$ are nondecreasing and left-continuous real-valued functions on the interval $I=[0, b)$ such that $R(0)=W(0)=0$. We define the corresponding inhomogeneous system.

Definition 2.6 Let $f \in \mathcal{L}^{2}(d W)$ and $\left[\begin{array}{ll}u_{1} & u_{2}\end{array}\right]^{T}$ be a vector-valued function such that the following equation

$$
\left[\begin{array}{l}
u_{1} \\
u_{2}
\end{array}\right](x)=\left[\begin{array}{l}
u_{1} \\
u_{2}
\end{array}\right](0)+\int_{0}^{x}\left[\begin{array}{cc}
0 & d R(t) \\
-d W(t) & 0
\end{array}\right]\left[\begin{array}{c}
f \\
u_{2}
\end{array}\right]
$$

holds for every point $x \in[0, b)$. The triple $\left(u_{1}, u_{2}, f\right)$ is said to belong to the set $\mathcal{T}$ if $u_{1} \in \mathcal{L}^{2}(d W)$.

Due to Remark 2.5 for every $\left(u_{1}, u_{2}, f\right) \in \mathcal{T}$ both functions $u_{1}$ and $u_{2}$ belong to $B V_{\text {loc }}[0, b)$. Theorem 2.4 implies that for every $f \in \mathcal{L}^{2}(d W)$ the vector-valued function $\left[\begin{array}{ll}u_{1} & u_{2}\end{array}\right]^{T}$ satisfying (2.17) is uniquely determined by its initial values at zero, however $u_{1} \in \mathcal{L}^{2}(d W)$ is not guaranteed for an arbitrary $f \in \mathcal{L}^{2}(d W)$.

Definition 2.7 We define the maximal and the pre-minimal relations $T_{\max }, T^{\prime} \subset$ $L^{2}(d W) \times L^{2}(d W)$ by

$$
T_{\max }:=\left\{\boldsymbol{u}=\left[\begin{array}{c}
\pi u_{1} \\
\pi f
\end{array}\right]:\left(u_{1}, u_{2}, f\right) \in \mathcal{T}\right\},
$$




$$
T^{\prime}:=\left\{\boldsymbol{u}=\left[\begin{array}{c}
\pi u_{1} \\
\pi f
\end{array}\right] \in T_{\max }:\left(u_{1}, u_{2}, f\right) \in \mathcal{T}, u_{1}, f \in \mathcal{L}_{\mathrm{comp}}^{2}(W, I)\right\} .
$$

where $\pi: \mathcal{L}^{2}(d W, I) \rightarrow L^{2}(d W, I)$ is the quotient map defined at the beginning of Sect. 2.3.

Denote $\mathfrak{N}_{\lambda}:=\mathfrak{N}_{\lambda}\left(T_{\max }\right), \lambda \in \mathbb{C} \backslash \mathbb{R}$. Everywhere in the paper, except Remark 3.10, we suppose that the following two natural assumptions hold.

Assumption 2.8 The functions $R$ and $W$ have no common points of discontinuity.

Assumption 2.9 There exists an interval $\left[0, b_{0}\right) \subseteq[0, b)$ such that

$$
\operatorname{dim} \operatorname{span}\{\pi 1, \pi R\}=2
$$

where $\pi: \mathcal{L}^{2}\left(W,\left[0, b_{0}\right)\right) \rightarrow L^{2}\left(W,\left[0, b_{0}\right)\right)$ is the corresponding quotient map.

Assumption 2.8 has the important consequence that the first component of a solution has no discontinuity in common with the second component of any solution $\left(u_{1}, u_{2}, f\right) \in \mathcal{T}$. Assumption 2.9 makes it possible to assign correctly the values $u_{1}(x)$ and $u_{2}(x)$ for every $\boldsymbol{u} \in T_{\max }$. In case of absolutely continuous functions $R$ and $W$ the differential system equivalent to $S[R, W]$ is definite in the sense of [36, Definition 2.14] if and only if Assumption 2.9 holds.

Definition 2.10 Let $\left(u_{1}, u_{2}, f\right) \in \mathcal{T}$ and $\boldsymbol{u} \in T_{\max }$ be its image under the mapping

$$
\mathcal{T} \ni\left(u_{1}, u_{2}, f\right) \mapsto \boldsymbol{u}=\left[\begin{array}{c}
\pi u_{1} \\
\pi f
\end{array}\right] \in T_{\max }
$$

The mappings $\phi_{1,2}[x]: T_{\max } \rightarrow \mathbb{C}$ are defined by

$$
\phi_{i}[x] \boldsymbol{u}:=u_{i}(x), \quad i \in\{1,2\}, \quad x \in[0, b) .
$$

The following Proposition provides a partial analog of [36, Proposition 2.15] and [12, Proposition 3.9] for the integral system $S[R, W]$.

Proposition 2.11 If Assumptions 2.8 and 2.9 hold then the mappings $\phi_{1,2}[x]$ are welldefined.

Proof Assume that $\left(u_{1}, u_{2}, f\right) \in \mathcal{T}$ and $\pi u_{1}=\pi f=0$. Let us show that under this assumption

$$
u_{1}(x)=u_{2}(x)=0 \text { for } x \in[0, b) .
$$

From the second line of (2.17) it follows immediately that

$$
u_{2}(x) \equiv u_{2}(0)
$$

Now substituting (2.23) in the first line of (2.17) we obtain

$$
u_{1}(x)=u_{1}(0)+u_{2}(0) R(x)
$$


The mapping $\pi$ applied to (2.24) gives

$$
0=u_{1}(0) \cdot \pi 1+u_{2}(0) \cdot \pi R \text {. }
$$

Now it follows from (2.20) that $u_{1}(0)=u_{2}(0)=0$, which together with (2.23) and (2.24) proves (2.22).

Further in the text we will simply write $u_{1,2}(x)$ instead of $\phi_{1,2}[x] \boldsymbol{u}$ unless this can lead to confusion. For a pair of vector-valued functions $u=\left[\begin{array}{ll}u_{1} & u_{2}\end{array}\right]^{T}, v=\left[\begin{array}{ll}v_{1} & v_{2}\end{array}\right]^{T}$ we define the generalized Wronskian by

$$
[u, v](x):=u_{1}(x) v_{2}(x)-u_{2}(x) v_{1}(x) .
$$

Proposition 2.12 If $\left(u_{1}, u_{2}, f\right)$ and $\left(v_{1}, v_{2}, g\right)$ belong to $\mathcal{T}$ then the following generalized first and second Green's identities hold

$$
\begin{aligned}
\int_{0}^{x} f v_{1} d W & =\int_{0}^{x} u_{2} v_{2} d R-u_{2}(x) v_{1}(x)+u_{2}(0) v_{1}(0), \\
\int_{0}^{x}\left(f v_{1}-u_{1} g\right) d W & =[u, v](x)-[u, v](0) .
\end{aligned}
$$

for an arbitrary interval $[0, x) \subset[0, b)$.

Proof We recall that due to Assumption 2.8 the functions $R$ and $W$ do not have common points of discontinuity, so neither do the functions $v_{1}$ and $u_{2}$. By virtue of (2.17) we get

$$
d v_{1}=v_{2} d R, \quad d u_{2}=-f d W .
$$

and hence, using the integration-by-parts formula (2.14):

$$
\int_{0}^{x} v_{1} d u_{2}+\int_{0}^{x} u_{2+} d v_{1}=u_{2}(x) v_{1}(x)-u_{2}(0) v_{1}(0)
$$

one obtains (2.26). Swapping the tuples $\left(u_{1}, u_{2}, f\right)$ and $\left(v_{1}, v_{2}, g\right)$ in (2.28) and subtracting the obtained expression from (2.26) proves (2.27).

Due to Theorem 2.4 the system $S[R, W]$ has a unique solution for every choice of initial values. Let $c(\cdot, \lambda)=\left[c_{1}(\cdot, \lambda) c_{2}(\cdot, \lambda)\right]^{T}$ and $s(\cdot, \lambda)=\left[s_{1}(\cdot, \lambda) s_{2}(\cdot, \lambda)\right]^{T}$ be its unique solutions satisfying the initial conditions (1.7).

Corollary 2.13 For every $\lambda \in \mathbb{C}$ and $x \in[0, b)$ the following formulas hold:

$$
\begin{aligned}
& {[c(\cdot, \lambda), s(\cdot, \lambda)](x)=c_{1}(x, \lambda) s_{2}(x, \lambda)-c_{2}(x, \lambda) s_{1}(x, \lambda)=1} \\
& c_{1+}(x, \lambda) s_{2}(x, \lambda)-c_{2}(x, \lambda) s_{1+}(x, \lambda)=1 \\
& c_{1}(x, \lambda) s_{2+}(x, \lambda)-c_{2+}(x, \lambda) s_{1}(x, \lambda)=1 .
\end{aligned}
$$


Proof Equality (2.29) follows immediately from (2.27). Further we subtract the lefthand side of (2.29) from the left-hand side of (2.30):

$$
\begin{aligned}
& \left(c_{1+}(x, \lambda) s_{2}(x, \lambda)-c_{2}(x, \lambda) s_{1+}(x, \lambda)\right)-\left(c_{1}(x, \lambda) s_{2}(x, \lambda)-c_{2}(x, \lambda) s_{1}(x, \lambda)\right) \\
& =\left(c_{1+}(x, \lambda)-c_{1}(x, \lambda)\right) s_{2}(x, \lambda)-c_{2}(x, \lambda)\left(s_{1+}(x, \lambda)-s_{1}(x, \lambda)\right)
\end{aligned}
$$

One can immediately see that the expression (2.32) is equal to zero at every point of continuity of $R$. Let $x_{0}$ be a point of discontinuity of $R$. From (2.17) one can see that

$$
\begin{aligned}
c_{1+}\left(x_{0}, \lambda\right)-c_{1}\left(x_{0}, \lambda\right) & =c_{2}\left(x_{0}, \lambda\right) d R\left(\left\{x_{0}\right\}\right), \\
s_{1+}\left(x_{0}, \lambda\right)-s_{1}\left(x_{0}, \lambda\right) & =s_{2}\left(x_{0}, \lambda\right) d R\left(\left\{x_{0}\right\}\right)
\end{aligned}
$$

and hence

$$
\begin{gathered}
\left(c_{1+}\left(x_{0}, \lambda\right)-c_{1}\left(x_{0}, \lambda\right)\right) s_{2}\left(x_{0}, \lambda\right)-c_{2}\left(x_{0}, \lambda\right)\left(s_{1+}\left(x_{0}, \lambda\right)-s_{1}\left(x_{0}, \lambda\right)\right) \\
=c_{2}\left(x_{0}, \lambda\right) s_{2}\left(x_{0}, \lambda\right) d R\left(\left\{x_{0}\right\}\right)-s_{2}\left(x_{0}, \lambda\right) c_{2}\left(x_{0}, \lambda\right) d R\left(\left\{x_{0}\right\}\right)=0 .
\end{gathered}
$$

The proof of (2.31) is similar.

It follows from (2.27) that the pre-minimal relation $T^{\prime}$ is symmetric in $L^{2}(d W)$.

Definition 2.14 The minimal relation $T_{\min }$ is defined as the closure of the pre-minimal linear relation $T^{\prime}: T_{\min }=\operatorname{clos} T^{\prime}$.

As was shown in [41] the linear relation $T_{\min }$ is symmetric, $T_{\min }^{*}=T_{\max }$ and

$$
T_{\min }:=\left\{\boldsymbol{u}=\left[\begin{array}{c}
\pi u_{1} \\
\pi f
\end{array}\right] \in T_{\max }: \begin{array}{l}
u_{1}(0)=0, \\
u_{2}(0)=0,
\end{array}[u, v]_{b}=0 \forall \boldsymbol{v}=\left[\begin{array}{c}
\pi v_{1} \\
\pi g
\end{array}\right] \in T_{\max }\right\} .
$$

Lemma 2.15 Let $l<b, h \in \operatorname{clos} \mathbb{C}_{+} \cup\{\infty\}$, and let $m(\lambda, l, h)$ be some coefficient such that the function

$$
\psi(t, \lambda):=s(t, \lambda)-m(\lambda, l, h) c(t, \lambda)
$$

satisfies the condition $\psi_{1}(l, \lambda)+h \psi_{2}(l, \lambda)=0$. Then:

(i) The coefficient $m$ is well-defined and can be calculated as

$$
m(\lambda, l, h)=\frac{s_{1}(l, \lambda)+h s_{2}(l, \lambda)}{c_{1}(l, \lambda)+h c_{2}(l, \lambda)} .
$$

(ii) For every $\lambda \in \mathbb{C}_{+}$the set $D_{l}(\lambda):=\left\{m(\lambda, l, h): h \in \operatorname{clos} \mathbb{C}_{+} \cup\{\infty\}\right\}$ is a disk in $\mathbb{C}_{+}$such that $\omega \in D_{l}(\lambda)$ if and only if

$$
\int_{0}^{l}\left|s_{1}(t, \lambda)-\omega c_{1}(t, \lambda)\right|^{2} d W(t) \leq \frac{\operatorname{Im} \omega}{\operatorname{Im} \lambda}
$$


and its radius can be calculated as

$$
r_{l}(\lambda)=\left(2 \operatorname{Im} \lambda \int_{0}^{l}\left|s_{1}(t, \lambda)\right|^{2} d W(t)\right)^{-1}
$$

(iii) The Weyl discs $D_{l}(\lambda)$ are nested, i.e. $D_{l_{2}} \subseteq D_{l_{1}}$ provided $l_{1}<l_{2}<b$, and the function $s_{1}(\cdot, \lambda)-\omega c_{1}(\cdot, \lambda)$ belongs to $\mathcal{L}^{2}(d W)$ provided $\omega \in \cap_{l<b} D_{l}(\lambda)$.

Proof (i) From (2.33) and the condition $\psi_{1}(l, \lambda)+h \psi_{2}(l, \lambda)=0$ we get

$$
\psi_{1}(l, \lambda)+h \psi_{2}(l, \lambda)=\left(s_{1}(l, \lambda)+h s_{2}(l, \lambda)\right)-m(\lambda, l, h)\left(c_{1}(l, \lambda)+h c_{2}(l, \lambda)\right)=0
$$

which yields (2.34).

(ii) It is clear from formula (2.34) that the function $m(\lambda, l, \cdot)$ maps $\mathbb{R}_{+} \cup\{\infty\}$ into a circle. Let $h \in \operatorname{clos} \mathbb{C}_{+} \cup\{\infty\}$ and $\omega:=m(\lambda, l, h) \in D_{l}(\lambda)$. Applying the second Green's identity (2.27) to the tuples $\left(\psi_{1}(\cdot, \lambda), \psi_{2}(\cdot, \lambda), \lambda \psi_{1}(\cdot, \lambda)\right)$ and $\left(\psi_{1}(\cdot, \bar{\lambda}), \psi_{2}(\cdot, \bar{\lambda}), \bar{\lambda} \psi_{1}(\cdot, \bar{\lambda})\right)$ provides

$$
(\lambda-\bar{\lambda}) \int_{0}^{l}\left|\psi_{1}(t, \lambda)\right|^{2} d R_{2}(t)=(\omega-\bar{\omega})-(h-\bar{h})\left|\psi_{2}(l, \lambda)\right|^{2}
$$

and hence

$$
\int_{0}^{l}\left|s_{1}(t, \lambda)-\omega c_{1}(t, \lambda)\right|^{2} d R_{2}(t)=\frac{\operatorname{Im} \omega}{\operatorname{Im} \lambda}-\frac{\operatorname{Im} h}{\operatorname{Im} \lambda}\left|\psi_{2}(l, \lambda)\right|^{2} .
$$

Since $\operatorname{Im} h \geq 0$, (2.35) follows now from (2.37).

(iii) The proof of (2.36) and item (iii) is standard, see [3, Section 8.13] and is omitted.

Assume that the point $b$ is singular for the system (1.1), i.e. $R(b)+W(b)=\infty$. Then the following alternative holds, [5, Proposition 2.4]:

(i) either discs $D_{l}(\lambda)$ shrink to a limit point as $l \rightarrow b$ for all $\lambda \in \mathbb{C} \backslash \mathbb{R}$ and then $\operatorname{dim} \mathfrak{N}_{\lambda}=1$ for all $\lambda \in \mathbb{C} \backslash \mathbb{R}$,

(ii) or discs $D_{l}(\lambda)$ converge to a limit disc as $l \rightarrow b$ for all $\lambda \in \mathbb{C} \backslash \mathbb{R}$ and then $\operatorname{dim} \mathfrak{N}_{\lambda}=2$ for all $\lambda \in \mathbb{C} \backslash \mathbb{R}$.

Definition 2.16 In the case (i) the system $S[R, W]$ is called limit point at $b$, in the case (ii) the system $S[R, W]$ is called limit circle at $b$.

Remark 2.17 1. A matrix version of an integral equation equivalent to the integral system $S[R, W]$ with $R(x) \equiv x$ and $W(x)$ continuous was considered in [2] and later in [39]. Such an equation can be reduced to a canonical differential system, see [2, Section 2.2]. Condition of definiteness of general matrix canonical differential system was found in [36]. In the scalar case this condition coincides with Assumption 2.9. 
2. Eckhardt and Teschl developed in [12] an operator approach to the Sturm-Liouville equation

$$
-\frac{d}{d W(x)}\left(\frac{d y}{d R(x)}+\int^{x} y(t) d Q(t)\right)=\lambda y(x), \quad x \in(a, b), \quad \lambda \in \mathbb{C},
$$

with measure coefficients $d W, d R$ and $d Q$ in the case when $R$ is strictly increasing. If in addition, $R$ and $W$ are continuous at $a=0$ integral system (1.1) is reduced to Eq. (2.38), where $Q \equiv 0$. However, in the case when $R$ is not strictly increasing the minimal relation $T_{\min }$ in Definition 2.14 may have a nontrivial multivalued part, which is not the case in [12]. For instance, if $W(x)=x, R(x)=(x-1) \chi_{(1,2)}(x)$, $x \in[0,2)$, then $\left(0, u_{2}, f\right) \in \mathcal{T}$ iff

$$
u_{2}(x)=-\int_{0}^{x} f(t) d t, \quad f \in L^{2}(d W,[0,1]) \ominus\{1\}
$$

and hence $\operatorname{mul}\left(T_{\min }\right)=L^{2}(d W,[0,1]) \ominus\{1\}$. Here $\chi_{(1,2)}(x)$ is the indicator of the interval $(1,2)$.

Differential systems with distributional coefficients were studied also recently in $[13,20]$.

\section{Integral Systems in the Limit Circle Case}

\subsection{The Fundamental Matrix of the System $S[R, W]$}

We will start with some general properties of the fundamental matrix of the system $S[R, W]$.

Lemma 3.1 Let $U(x, \lambda)$ be the fundamental matrix function of the system $S[R, W]$

$$
U(x, \lambda):=\left[\begin{array}{ll}
c_{1}(x, \lambda) & s_{1}(x, \lambda) \\
c_{2}(x, \lambda) & s_{2}(x, \lambda)
\end{array}\right], \quad \lambda \in \mathbb{C} .
$$

Then:

(i) For every $\lambda, \mu \in \mathbb{C}$ the following identity holds

$$
J-U(x, \mu)^{*} J U(x, \lambda)=-(\lambda-\bar{\mu}) \int_{0}^{x}\left[\begin{array}{l}
c_{1}(t, \bar{\mu}) \\
s_{1}(t, \bar{\mu})
\end{array}\right]\left[c_{1}(t, \lambda) s_{1}(t, \lambda)\right] d W(t) .
$$

(ii) For every $x \in[0, b), U(x, \lambda)$ is entire in $\lambda$.

(iii) The entries of $U(x, \lambda)$ are nonnegative for $x \in[0, b), \lambda \in \mathbb{R}_{-}$. If, in addition, the interval $(0, x)$ contains growth points of $R$ and $W$, and

$$
a=\inf \operatorname{supp} d W, \quad a_{1}=\inf (\operatorname{supp} d R \cap(a, b)),
$$


then

$$
\begin{aligned}
& \lim _{\lambda \rightarrow-\infty} c_{1}(x, \lambda)=+\infty, x \in\left(a_{1}, b\right) ; \quad \lim _{\lambda \rightarrow-\infty} c_{2}(x, \lambda)=+\infty, x \in(a, b) ; \\
& \lim _{\lambda \rightarrow-\infty} s_{1}(x, \lambda)=+\infty, x \in\left(a_{1}, b\right) ; \quad \lim _{\lambda \rightarrow-\infty} s_{2}(x, \lambda)=+\infty, x \in(a, b) .
\end{aligned}
$$

(iv) If $\lambda \in \mathbb{R}_{-}$then

$$
\frac{s_{1}(x, \lambda)}{c_{1}(x, \lambda)}<\frac{s_{2}(x, \lambda)}{c_{2}(x, \lambda)}, \quad x \in(a, b)
$$

the function $\frac{s_{1}(x, \lambda)}{c_{1}(x, \lambda)}$ is increasing on $[0, b)$ and the function $\frac{s_{2}(x, \lambda)}{c_{2}(x, \lambda)}$ is decreasing on $(a, b)$.

Proof 1. By (2.26) for the triples $\left(c_{1}(\cdot, \lambda), c_{2}(\cdot, \lambda), \lambda c_{1}(\cdot, \lambda)\right) \in \mathcal{T}$ and $\left(c_{1}(\cdot, \mu), c_{2}(\cdot, \mu), \mu c_{1}(\cdot, \mu)\right) \in \mathcal{T}$ one obtains

$$
(\lambda-\bar{\mu}) \int_{0}^{x} c_{1}(t, \lambda) c_{1}(t, \bar{\mu}) d W=c_{1}(x, \lambda) c_{2}(x, \bar{\mu})-c_{2}(x, \lambda) c_{1}(x, \bar{\mu}) .
$$

this proves (i) for the 1, 1-blocks of (3.2).

The proof for other blocks of (3.2) is similar.

2. It follows from (3.2) that

$$
U(x, \mu)^{*}=J U(x, \bar{\mu})^{-1} J^{T}, \quad \mu \in \mathbb{C} .
$$

Therefore,

$$
\frac{U(x, \lambda)-U(x, \bar{\mu})}{\lambda-\bar{\mu}}=U(x, \bar{\mu}) J^{T} \int_{0}^{x}\left[\begin{array}{l}
c_{1}(t, \bar{\mu}) \\
s_{1}(t, \bar{\mu})
\end{array}\right]\left[c_{1}(t, \lambda) s_{1}(t, \lambda)\right] d W(t),
$$

hence $U(x, \lambda)$ is holomorphic on $\mathbb{C}$ which proves (ii).

3 . To show (iii), expanding $c_{1}(x, \lambda)$ and $c_{2}(x, \lambda)$ in series in $\lambda$

$$
c_{1}(x, \lambda)=1-\lambda \varphi_{1}(x)+\lambda^{2} \varphi_{2}(x)+\cdots, \quad c_{2}(x, \lambda)=-\lambda \psi_{1}(x)+\lambda^{2} \psi_{2}(x)+\cdots
$$

one obtains from (1.1) that

$$
\begin{aligned}
& \psi_{1}(x)=W(x), \quad \varphi_{1}(x)=\int_{0}^{x} W(t) d R(t) \\
& \psi_{n}(x)=\int_{0}^{x} \varphi_{n-1}(t) d W(t), \quad \varphi_{n}(x)=\int_{0}^{x} d R(t) \int_{0}^{t} \varphi_{n-1}(s) d W(s), \quad n \in \mathbb{N} .
\end{aligned}
$$


This implies that $\varphi_{n}(x) \geq 0, \psi_{n}(x) \geq 0$ for $n \in \mathbb{N}$ and hence

$$
c_{1}(x, \lambda) \geq 0, \quad c_{2}(x, \lambda) \geq 0 \text { for } x \in[0, b), \lambda \in \mathbb{R}_{-} .
$$

Moreover, it follows from (3.8) that

$$
c_{1}(x, \lambda) \geq 1+|\lambda| \int_{0}^{x} W(t) d R(t), \quad c_{2}(x, \lambda) \geq|\lambda| W(x) .
$$

Therefore, the relations (3.4) hold since

$$
\int_{0}^{x} W(t) d R(t)>0 \text { for } x \in\left(a_{1}, b\right) \text { and } W(x)>0 \text { for } x \in(a, b)
$$

The proof of (3.5) is similar.

4. The identity (2.29) yields

$$
\frac{s_{2}(x, \lambda)}{c_{2}(x, \lambda)}-\frac{s_{1}(x, \lambda)}{c_{1}(x, \lambda)}=\frac{1}{c_{1}(x, \lambda) c_{2}(x, \lambda)}
$$

This proves the inequality (3.6).

It follows from (1.1), (2.14), (2.15), and (2.30) that

$$
\begin{aligned}
d\left(\frac{s_{1}(x, \lambda)}{c_{1}(x, \lambda)}\right) & =\frac{c_{1+}(x, \lambda) s_{2}(x, \lambda)-c_{2}(x, \lambda) s_{1+}(x, \lambda)}{c_{1}(x, \lambda) c_{1+}(x, \lambda)} d R(x) \\
& =\frac{1}{c_{1}(x, \lambda) c_{1+}(x, \lambda)} d R(x)
\end{aligned}
$$

and hence

$$
\frac{s_{1}(x, \lambda)}{c_{1}(x, \lambda)}=\int_{0}^{x} \frac{1}{c_{1}(t, \lambda) c_{1+}(t, \lambda)} d R(t)
$$

Since $c_{1}(x, \lambda), c_{1+}(x, \lambda)>0$ for $\lambda \in \mathbb{R}_{-}$and $x \in[0, b)$, the function $\frac{s_{1}(x, \lambda)}{c_{1}(x, \lambda)}$ is increasing on $[0, b)$.

Similarly, by (1.1), (2.14), (2.15), and (2.31)

$$
d\left(\frac{c_{2}(x, \lambda)}{s_{2}(x, \lambda)}\right)=\frac{-\lambda}{s_{2}(x, \lambda) s_{2+}(x, \lambda)} d W(x), \quad x \in[0, b)
$$

and hence the function $\frac{c_{2}(x, \lambda)}{s_{2}(x, \lambda)}$ is increasing on $[0, b)$. This proves (iv). Notice, that the function $\frac{s_{2}(x, \lambda)}{c_{2}(x, \lambda)}$ is not defined on $[0, a]$.

\subsection{The Evans-Everitt Condition in the Limit Circle Case}

Proposition 3.2 The system $S[R, W]$ is limit circle at $b$ if and only if $1, R \in \mathcal{L}^{2}(d W)$. 
Proof Using the well-known procedure from [3, Theorem 5.6.1] (see also [41, Theorem 4.5]) one can show that $S[R, W]$ is limit circle at $b$ if and only if $c_{1}(x, 0)$ and $s_{1}(x, 0)$ belong to $\mathcal{L}^{2}(d W)$. Substitution of $\lambda=0$ to (1.1) gives $c_{2}(x, 0)=0$, $s_{2}(x, 0)=1$ and hence $c_{1}(x, 0)=1, s_{1}(x, 0)=R(x)$.

If the system $S[R, W]$ is regular at $b$, then the following limits exist:

$$
\begin{array}{ll}
c_{1}(b, \lambda)=\lim _{t \rightarrow b} c_{1}(t, \lambda), & s_{1}(b, \lambda)=\lim _{t \rightarrow b} s_{1}(t, \lambda), \\
c_{2}(b, \lambda)=\lim _{t \rightarrow b} c_{2}(t, \lambda), & s_{2}(b, \lambda)=\lim _{t \rightarrow b} s_{2}(t, \lambda) .
\end{array}
$$

Assume now that the system $S[R, W]$ is limit circle at $b$. One can check (see [27, Section 10.7], [40, Theorem 3.8]) that for every $\boldsymbol{u}=\left[\begin{array}{c}\pi u_{1} \\ \pi f\end{array}\right] \in T_{\max }$ the limit

$$
u_{2}(b)=u_{2}(0)-\int_{0}^{b} f d W
$$

exists and is well defined. Therefore, the limits (3.14) exist.

Consider a one-dimensional symmetric extension $T_{N}$ of the linear relation $T_{\min }$ defined by

$$
T_{N}=\left\{\boldsymbol{u}=\left[\begin{array}{c}
\pi u_{1} \\
\pi f
\end{array}\right]:\left(u_{1}, u_{2}, f\right) \in \mathcal{T}, u_{1}(0)=u_{2}(0)=u_{2}(b)=0\right\} .
$$

As follows from (2.27) the adjoint linear relation $T_{N}^{*}$ is of the form

$$
T_{N}^{*}=\left\{\boldsymbol{u}=\left[\begin{array}{c}
\pi u_{1} \\
\pi f
\end{array}\right]:\left(u_{1}, u_{2}, f\right) \in \mathcal{T}: u_{2}(b)=0\right\} .
$$

Lemma 3.3 Let the system $S[R, W]$ be limit circle at $b$. Then for every $\boldsymbol{u}=\left[\begin{array}{c}\pi u_{1} \\ \pi f\end{array}\right] \in$ $T_{N}^{*}$ one has $u_{2} \in \mathcal{L}^{2}(R)$ and the following two equalities hold:

$$
\begin{aligned}
& \lim _{x \rightarrow b} u_{1}(x)=u_{1}(0)+\langle f, R\rangle, \\
& \lim _{x \rightarrow b} u_{1}(x) u_{2}(x)=0 .
\end{aligned}
$$

Conversely, if $\boldsymbol{u} \in T_{\max }$, the endpoint $b$ is singular and (3.19) holds, then $\boldsymbol{u} \in T_{N}^{*}$.

Proof Let $\boldsymbol{u}=\left[\begin{array}{c}\pi u_{1} \\ \pi f\end{array}\right] \in T_{N}^{*}$. Applying the integration-by-parts formula (2.14) to the first line of (2.17) one gets

$$
u_{1}(x)=u_{1}(0)+u_{2}(x) R(x)+\int_{0}^{x} R(t) f(t) d W(t) .
$$


We recall that in the limit circle case $1, R \in \mathcal{L}^{2}(d W)$ and $f \in \mathcal{L}^{2}(d W)$ by the assumption of the lemma. The condition $u_{2}(b)=0$ implies that $u_{2}(x)=\int_{x}^{b} f d W$ and hence (3.20) can be rewritten as

$$
u_{1}(x)=u_{1}(0)+\langle f, R\rangle-\int_{x}^{b}(R(t)-R(x)) f(t) d W(t) .
$$

Note the following estimation:

$$
\begin{aligned}
\left|\int_{x}^{b}(R(t)-R(x)) f(t) d W(t)\right| & \leq \int_{x}^{b}(R(t)-R(x))|f(t)| d W(t) \\
& \leq \int_{x}^{b} R|f| d W \rightarrow 0 \text { as } x \rightarrow b .
\end{aligned}
$$

Now (3.18) follows from (3.21) and (3.22), and (3.19) finally follows from (3.18).

The claim $u_{2} \in \mathcal{L}^{2}(R)$ for $\boldsymbol{u}=\left[\begin{array}{c}\pi u_{1} \\ \pi f\end{array}\right] \in T_{N}^{*}$ follows from (3.18) and the first Green's identity (2.26)

$$
\begin{aligned}
\int_{0}^{b} f(t) \overline{u_{1}(t)} d W(t) & =\int_{0}^{b}\left|u_{2}\right|^{2} d R(t)-\lim _{x \rightarrow b} u_{2}(x) \overline{u_{1}(x)}+u_{2}(0) \overline{u_{1}(0)} \\
& =\int_{0}^{b}\left|u_{2}\right|^{2} d R(t)+u_{2}(0) \overline{u_{1}(0)}
\end{aligned}
$$

Now assume that the endpoint $b$ is singular and $\boldsymbol{u}=\left[\begin{array}{c}\pi u_{1} \\ \pi f\end{array}\right] \in T_{\max }$. From (3.15) we have $u_{2}(b)=a$ where $a \in \mathbb{C}$. In the limit circle case the singular endpoint $b$ implies $R(b)=\infty$. If $a \neq 0$ then from (2.17) we get $u_{1}(b)= \pm \infty$ and hence (3.19) does not hold.

Remark 3.4 The condition (3.19) for Sturm-Liouville operators in the limit circle case was introduced and studied by Evans and Everitt in [17]. We will call it the EvansEveritt condition.

\subsection{Boundary Triples for Integral Systems in the Limit Circle Case}

Definition 3.5 (see [5,33]) The function $m(\lambda, b, \infty)$ from $(2.33)$ for which the solution

$$
\psi^{N}(t, \lambda)=s(t, \lambda)-m(\lambda, b, \infty) c(t, \lambda), \quad t \in I,
$$

satisfies the condition

$$
\psi_{2}^{N}(b, \lambda)=0
$$

is called the Neumann m-function of the system $S[R, W]$ on $I$ subject to the boundary condition (3.25) and $\psi^{N}(t, \lambda)$ is called the Weyl solution of (1.1). 
It follows from (2.33) and the condition $\psi_{2}^{N}(b, \lambda)=0$ that $s_{2}(b, \lambda)-$ $m(\lambda, b, \infty) c_{2}(b, \lambda)=0$ which proves the formula

$$
m(\lambda, b, \infty)=\frac{s_{2}(b, \lambda)}{c_{2}(b, \lambda)} .
$$

We will show below that the function $m(\lambda, b, \infty)$ is the Weyl function of the linear relation $T_{N}$ in the sense of Definition 2.2.

Proposition 3.6 Let the system $S[R, W]$ be singular and limit circle at $b$, let $T_{N}$ be defined by (3.16), and let $m(\lambda, b, \infty)$ be the Neumann $m$-function of the system $S[R, W]$ given by (3.26). Then:

(i) $T_{N}$ is a symmetric nonnegative linear relation in $L^{2}(d W)$ with deficiency indices $(1,1)$.

(ii) The triple $\Pi^{N}=\left(\mathbb{C}, \Gamma_{0}^{N}, \Gamma_{1}^{N}\right)$, where

$$
\Gamma_{0}^{N} \boldsymbol{u}=u_{2}(0), \quad \Gamma_{1}^{N} \boldsymbol{u}=-u_{1}(0), \quad \boldsymbol{u} \in T_{N}^{*},
$$

is a boundary triple for $T_{N}^{*}$.

(iii) The Weyl function $m_{N}(\lambda)$ of $T_{N}$ corresponding to the boundary triple $\Pi^{N}$ coincides with the Neumann $m$-function $m(\lambda, b, \infty)$.

(iv) The Weyl function $m_{N}(\lambda)$ of $T_{N}$ coincides with the principal Titchmarsh-Weyl coefficient $q(\lambda)$ of the system $S[R, W]$ defined in (1.6), belongs to the Stieltjes class $\mathcal{S}$, and

$$
\lim _{\lambda \rightarrow-\infty} m_{N}(\lambda)=R_{+}(a)
$$

where $a=\inf \operatorname{supp} d W$.

(v) The Weyl function $m_{N}(\lambda)$ of $T_{N}$ admits the representation

$$
m_{N}(\lambda)=-\frac{1}{W(b) \cdot \lambda}+\tilde{m}(\lambda)
$$

where $\tilde{m}$ is a function from $\mathcal{S}$ such that $\lim _{y \rightarrow 0} y \tilde{m}(i y)=0$.

Proof 1. To show (i), (ii), let the tuples $\left(u_{1}, u_{2}, f\right),\left(v_{1}, v_{2}, g\right) \in \mathcal{T}$ satisfy $u_{2}(b)=$ $v_{2}(b)=0$, i.e. $\boldsymbol{u}, \boldsymbol{v} \in T_{N}^{*}$. Let $\mu \in \mathbb{R}$. By formula (2.29) at least one of the values $c_{2}(b, \mu)$ and $s_{2}(b, \mu)$ is not equal to 0 . Assume that $c_{2}(b, \mu) \neq 0$. Due to the identity

$$
[u, v](b)=c_{2}(b, \mu)^{-1}\left\{[u(\cdot), c(\cdot, \mu)](b) \overline{v_{2}(b)}-u_{2}(b)[\overline{v(\cdot)}, c(\cdot, \mu)](b)\right\}
$$

the second Green's identity (2.27) is of the form

$$
\int_{0}^{b}\left(f \overline{v_{1}}-u_{1} \bar{g}\right) d W(t)=[u, \bar{v}](b)-[u, \bar{v}](0)=u_{2}(0) \overline{v_{1}(0)}-u_{1}(0) \overline{v_{2}(0)}
$$

By Definition 2.1 the boundary triple for $T_{N}^{*}$ can be taken as $\Pi^{N}=\left(\mathbb{C}, \Gamma_{0}^{N}, \Gamma_{1}^{N}\right)$, with $\Gamma_{0}^{N}, \Gamma_{1}^{N}$ given in (3.27). 
It follows from the first Green's identity (3.23) and Lemma 3.3 that for every $\left(\pi u_{1}, \pi f\right)^{T} \in T_{N}$

$$
\int_{0}^{b} f(t) \overline{u_{1}(t)} d W(t)=\int_{0}^{b}\left|u_{2}\right|^{2} d R(t) \geq 0 .
$$

2. Let us prove (iii). The defect subspace $\mathfrak{N}_{\lambda}\left(T_{N}^{*}\right)$ is spanned by the function $\psi_{1}^{N}(\cdot, \lambda)$, where $\psi^{N}(t, \lambda)$ is the Weyl solution from (3.24). Denote $\boldsymbol{u}^{N}(t, \lambda)=$ $\left(\psi_{1}^{N}(\cdot, \lambda), \lambda \psi_{1}^{N}(\cdot, \lambda)\right)^{T} \in \widehat{\mathfrak{N}}_{\lambda}\left(T_{N}^{*}\right)$. Using (3.24), (3.27) one obtains

$$
\Gamma_{1}^{N} \boldsymbol{u}^{N}(\cdot, \lambda)=-\psi_{1}^{N}(0, \lambda)=m(\lambda, b, \infty), \quad \Gamma_{0}^{N} \boldsymbol{u}^{N}(\cdot, \lambda)=\psi_{2}^{N}(0, \lambda)=1
$$

and hence by (2.10) the Weyl function $m_{N}(\lambda)$ is of the form

$$
m_{N}(\lambda)=\frac{\Gamma_{1}^{N} \boldsymbol{u}^{N}(\cdot, \lambda)}{\Gamma_{0}^{N} \boldsymbol{u}^{N}(\cdot, \lambda)}=m(\lambda, b, \infty) .
$$

Therefore, the Weyl function $m_{N}(\lambda)$ coincides with the Neumann $m$-function $m(\lambda, b, \infty)$.

3. The inclusion $m_{N} \in \mathcal{S}$ follows from Lemma 3.1, since the functions $s_{2}(x, \lambda)$ and $c_{2}(x, \lambda)$ are positive for $\lambda<0$ and the function $m_{N}(\lambda)$ admits a holomorphic nonnegative continuation on $\mathbb{R}_{-}$.

Let $a=\inf \operatorname{supp} W$ and $a_{1}=\inf (\operatorname{supp} R \cap(a, b))$. Then by Assumption $2.9 a_{1}<b$ and due to (1.1) and Lemma 3.1 (iii)

$$
c_{1}(x, \lambda) \equiv 1 \text { for } x \leq a_{1} \text { and } \lim _{\lambda \rightarrow-\infty} c_{1}(x, \lambda)=+\infty \text { for } x>a_{1}
$$

Now we must consider two cases:

(a) $a_{1}>a$ and $R$ has a jump at $a_{1}$;

(b) either $a_{1}=a$ or $a_{1}>a$ and $R$ has no jump at $a_{1}$.

In case (a) $c_{1}(\cdot, \lambda)$ has a jump at point $a_{1}$ and we get

$$
\frac{1}{c_{1}(x, \lambda) c_{1+}(x, \lambda)} \rightarrow \chi_{\left[0, a_{1}\right)}(x) \text { as } \lambda \rightarrow-\infty
$$

and hence by the Lebesgue bounded convergence theorem one obtains from (3.11)

$$
\lim _{\lambda \rightarrow-\infty} \frac{s_{1}(x, \lambda)}{c_{1}(x, \lambda)}=\int_{0}^{x} \frac{d R(t)}{c_{1}(t, \lambda) c_{1+}(t, \lambda)}=\int_{\left[0, a_{1}\right)} d R=R\left(a_{1}\right)=R_{+}(a) .
$$

The last equality in (3.34) follows from $a_{1}>a$ and (3.3).

In case (b) $c_{1}(\cdot, \lambda)$ has no jump at point $a_{1}$ and we get

$$
\frac{1}{c_{1}(x, \lambda) c_{1+}(x, \lambda)} \rightarrow \chi_{\left[0, a_{1}\right]}(x) \quad \text { as } \quad \lambda \rightarrow-\infty .
$$


Similarly to (3.34) one obtains

$$
\lim _{\lambda \rightarrow-\infty} \frac{s_{1}(x, \lambda)}{c_{1}(x, \lambda)}=R_{+}\left(a_{1}\right)=R_{+}(a)
$$

Since $R(b)+W(b)=+\infty$ it follows from (3.9) that $\lim _{x \rightarrow b} c_{1}(x, \lambda) c_{2}(x, \lambda)=+\infty$ for all $\lambda \in \mathbb{R}_{-}$and hence it follows from (3.10) that

$$
q(\lambda)=\lim _{x \rightarrow b} \frac{s_{1}(x, \lambda)}{c_{1}(x, \lambda)}=\lim _{x \rightarrow b} \frac{s_{2}(x, \lambda)}{c_{2}(x, \lambda)}=m_{N}(\lambda), \quad \lambda \in \mathbb{R}_{-} .
$$

Since $q$ and $m_{N}$ are holomorphic on $\mathbb{C} \backslash \mathbb{R}_{+}$this proves that $q(\lambda) \equiv m_{N}(\lambda)$, and (iv) is shown.

4. Now we prove (v). It follows from (1.1) and (3.1) that

$$
s_{2}(x, \lambda)=1-\lambda \int_{0}^{x} s_{1}(t, \lambda) d W(t), \quad c_{2}(x, \lambda)=-\lambda \int_{0}^{x} c_{1}(t, \lambda) d W(t)
$$

and by (3.26) that

$$
m_{N}(\lambda)=\frac{1-\lambda \int_{0}^{b} s_{1}(t, \lambda) d W(t)}{-\lambda \int_{0}^{b} c_{1}(t, \lambda) d W(t)}, \quad \lambda \in \mathbb{C} \backslash \mathbb{R}
$$

Moreover, for $\lambda<0$ the functions $s_{1}(x, \lambda)$ and $c_{1}(x, \lambda)$ are positive and increasing on $(0, b)$ and $c_{2}(0, \lambda)=1$, hence

$$
\int_{0}^{b} c_{1}(t, \lambda) d W(t)>W(b), \quad \int_{0}^{b} s_{1}(t, \lambda) d W(t)>0 .
$$

Since $c_{1}(x, \lambda) \rightarrow c_{1}(x, 0) \equiv 1$ and $s_{1}(x, \lambda) \rightarrow s_{1}(x, 0)=R(x)$ as $\lambda \rightarrow 0-$ and these convergences are monotone and uniform on $[0, b]$ one finds that

$$
\int_{0}^{b} c_{1}(t, \lambda) d W(t) \rightarrow W(b), \quad \int_{0}^{b} s_{1}(t, \lambda) d W(t) \rightarrow \int_{0}^{b} R(t) d W(t)
$$

as $\lambda \rightarrow 0-$. Therefore,

$$
\lambda m_{N}(\lambda) \rightarrow-\frac{1}{W(b)}, \quad \text { as } \quad \lambda \rightarrow 0-
$$

and thus $m_{N}(\lambda)$ admits the representation (3.29). 


\subsection{Integral Systems in the Regular Case}

Assume that the system $S[R, W]$ is regular at $b$, i.e. $R(b)+W(b)<\infty$. Then for every tuple $\left(u_{1}, u_{2}, f\right) \in \mathcal{T}$ it follows from (3.15) that the function $u_{2}$ is bounded and hence the limit

$$
u_{1}(b)=u_{1}(0)+\int_{0}^{b} u_{2} d R
$$

exists and is well defined. Therefore, the limits (3.13) exist.

Definition 3.7 (see [5,33]) The function $m(\lambda, b, 0)$ for which the solution

$$
\psi^{N D}(t, \lambda)=s(t, \lambda)-m(\lambda, b, 0) c(t, \lambda), \quad t \in I,
$$

satisfies the condition

$$
\psi_{1}^{N D}(b, \lambda)=0
$$

is called the Neumann m-function of the system $S[R, W]$ on $I$ subject to the boundary condition (3.42).

It follows from (2.33) and the condition $\psi_{1}^{N D}(b, \lambda)=0$ that $s_{1}(b, \lambda)-m(\lambda, b, 0) c_{1}(b, \lambda)=$ 0 which yields the formula

$$
m(\lambda, b, 0)=\frac{s_{1}(b, \lambda)}{c_{1}(b, \lambda)}
$$

and hence the Neumann $m$-function $m(\lambda, b, 0)$ coincides with the principal TitchmarshWeyl coefficient $q(\lambda)$ of the system $S[R, W]$, defined in (1.6).

Let $T_{D}$ be a symmetric extension of the linear relation $T_{\min }$ defined by

$$
T_{D}=\left\{\boldsymbol{u}=\left[\begin{array}{c}
\pi u_{1} \\
\pi f
\end{array}\right]:\left(u_{1}, u_{2}, f\right) \in \mathcal{T}, u_{1}(0)=u_{2}(0)=u_{1}(b)=0\right\} .
$$

As follows from (2.27) the adjoint linear relation $T_{D}^{*}$ is of the form

$$
T_{D}^{*}=\left\{\boldsymbol{u}=\left[\begin{array}{c}
\pi u_{1} \\
\pi f
\end{array}\right]:\left(u_{1}, u_{2}, f\right) \in \mathcal{T}: u_{1}(b)=0\right\} .
$$

Proposition 3.8 (cf. [40]) Let the system $S[R, W]$ be regular at $b$, and let $T_{D}$ be defined by (3.44). Then:

(i) $T_{D}$ is a symmetric nonnegative linear relation in $L^{2}(d W)$ with deficiency indices $(1,1)$ and $u_{2} \in L^{2}(R)$ for all $\boldsymbol{u}=\left[\begin{array}{c}\pi u_{1} \\ \pi f\end{array}\right] \in T_{D}^{*}$

(ii) the triple $\Pi^{N D}=\left(\mathbb{C}, \Gamma_{0}^{N D}, \Gamma_{1}^{N D}\right)$, where

$$
\Gamma_{0}^{N D} \boldsymbol{u}=u_{2}(0), \quad \Gamma_{1}^{N D} \boldsymbol{u}=-u_{1}(0), \quad \boldsymbol{u} \in T_{D}^{*},
$$

is a boundary triple for $T_{D}^{*}$. 
(iii) The Weyl function $m_{N D}(\lambda)$ of $T_{D}$ corresponding to the boundary triple $\Pi^{N D}$ coincides with $m(\lambda, b, 0)$.

(iv) The Weyl function $m_{N D}(\lambda)$ of $T_{D}$ belongs to the Stieltjes class $\mathcal{S}$ and coincides with the principal Titchmarsh-Weyl coefficient $q(\lambda)$ of the system $S[R, W]$.

Proof 1. To show (i) and (ii), let the tuples $\left(u_{1}, u_{2}, f\right)$ and $\left(v_{1}, v_{2}, g\right)$ satisfy the system (2.17) and assume that $u_{1}(b)=v_{1}(b)=0$, i.e. $\boldsymbol{u}, \boldsymbol{v} \in T_{D}^{*}$. Let $\mu \in \mathbb{R}$. By (2.29) at least one of the values $c_{1}(b, \mu)$ and $s_{1}(b, \mu)$ is not equal to 0 . Assume that $c_{1}(b, \mu) \neq 0$. Due to the identity

$$
[u, v](b)=c_{1}(b, \mu)^{-1}\left\{[u(\cdot), c(\cdot, \mu)](b) \overline{v_{1}(b)}-u_{1}(b)[\overline{v(\cdot)}, c(\cdot, \mu)](b)\right\}
$$

the Green's identity (2.27) is of the form (3.30). By Definition 2.1 the boundary triple for $T_{D}^{*}$ can be taken as $\Pi^{N D}=\left(\mathbb{C}, \Gamma_{0}^{N D}, \Gamma_{1}^{N D}\right)$, with $\Gamma_{0}^{N D}, \Gamma_{1}^{N D}$ given in (3.46).

It follows from the first Green's identity (2.26) and Lemma 3.3 that for every $\boldsymbol{u} \in T_{D}$ the identity (3.31) holds and thus the linear relation $T_{D}$ is nonnegative.

2. Let us prove (iii). The defect subspace $\mathfrak{N}_{\lambda}\left(T_{D}\right)$ is spanned by the function $\psi_{1}^{N D}(\cdot, \lambda)$ determined by (3.41). Denote

$$
\boldsymbol{u}^{N D}(t, \lambda)=\left(\psi_{1}^{N D}(\cdot, \lambda), \lambda \psi_{1}^{N D}(\cdot, \lambda)\right)^{T} \in \widehat{\mathfrak{N}}_{\lambda}\left(T_{D}^{*}\right)
$$

Using the formulae (3.41) and (1.7) one obtains

$$
\Gamma_{1}^{N D} \boldsymbol{u}^{N D}(\cdot, \lambda)=-\psi_{1}^{N D}(0, \lambda)=m(\lambda, b, 0), \quad \Gamma_{0}^{N D} \boldsymbol{u}^{N D}(\cdot, \lambda)=\psi_{2}^{N D}(0, \lambda)=1
$$

and hence the Weyl function $m_{N D}(\lambda)$ is of the form

$$
m_{N D}(\lambda)=\frac{\Gamma_{1}^{N D} \boldsymbol{u}^{N D}(\cdot, \lambda)}{\Gamma_{0}^{N D} \boldsymbol{u}^{N D}(\cdot, \lambda)}=m(\lambda, b, 0)
$$

Therefore, the Weyl function $m_{N D}(\lambda)$ coincides with the Neumann $m$-function $m(\lambda, b, 0)$.

3. Finally we prove (iv). The inclusion $m_{N D} \in \mathcal{S}$ follows from Lemma 3.1. The equality $m_{N D}(\lambda) \equiv q(\lambda), \lambda \in \mathbb{C} \backslash \mathbb{R}$, is implied by (3.43).

Remark 3.9 The functions $R$ and $W$ are not uniquely defined by the principal Titchmarsh-Weyl coefficient of the system $S[R, W]$. As was shown in [33, Lemma 2.12] if functions $\widetilde{R}(\xi)$ and $\widetilde{W}(\xi)$ are connected by

$$
\widetilde{R}(\xi)=R(x(\xi)), \quad \widetilde{W}(\xi)=W(x(\xi)), \quad \xi \in[0, \beta] .
$$

where $x(\xi)$ is an increasing function on the interval $[0, \beta]$, such that $x(0)=0$ and $x(\beta)=b$, then the principal Titchmarsh-Weyl coefficient $\widetilde{q}$ of the system

$$
\tilde{u}(\xi, \lambda)=\widetilde{u}(0, \lambda)-J \int_{0}^{\xi}\left[\begin{array}{cc}
\lambda d \widetilde{W}(\tau) & 0 \\
0 & d \widetilde{R}(\tau)
\end{array}\right] \widetilde{u}(\tau, \lambda), \quad \xi \in[0, \beta] .
$$


coincides with the principal Titchmarsh-Weyl coefficient $q$ of the system $S[R, W]$.

Therefore we can always assume that for regular systems $S[R, W]$ the parameter $x$ ranges over a finite interval $[0, b], b<\infty$.

Remark 3.10 As is known, see [27, Section A13], a truncated moment problem can be reduced to a regular integral system $S[R, W]$ with

$$
\begin{aligned}
R(x) & =x, \quad W(x)=\sum_{j=0}^{n-1} m_{j} H\left(x-x_{j}\right), \quad x \in\left[0, x_{n}\right], \\
x_{j} & =\sum_{j=1}^{j} l_{i}, \quad m_{j-1}, l_{j}>0, \quad 1 \leq j \leq n .
\end{aligned}
$$

where $H(x)$ is the Heaviside function. The corresponding monodromy matrix $U\left(x_{n}, \lambda\right)$ is of the form

$$
U\left(x_{n}, \lambda\right)=\prod_{j=1}^{n} U_{x_{j-1}}\left(x_{j}, \lambda\right), \quad \text { where } \quad U_{x_{j-1}}\left(x_{j}, \lambda\right)=\left[\begin{array}{cc}
1-\lambda l_{j} m_{j-1} & l_{j} \\
-\lambda m_{j-1} & 1
\end{array}\right] .
$$

The system $S[R, W]$ satisfies Assumption 2.9 if $n>1$. If $n=1$ then $W(x)=H(x)$, $x \in\left[0, l_{1}\right], L^{2}(d W)=\mathbb{C}$, the system $S[R, W]$ is of the form

$$
u_{1}(x)=u_{1}(0)+x u_{2}(x), \quad u_{2}(x)=u_{2}(0)-\lambda u_{1}(0) m_{0}, \quad x \in\left(0, l_{1}\right]
$$

and does not satisfy the Assumption 2.9. However, in this case one can still introduce a boundary triple $\left(\mathbb{C}, \Gamma_{0}, \Gamma_{1}\right)$ for $T_{\max }=\mathbb{C} \times \mathbb{C}$ by

$$
\Gamma_{0} \boldsymbol{u}=u_{1}(0), \quad \Gamma_{1} \boldsymbol{u}=f(0), \quad \boldsymbol{u}=\left[\begin{array}{c}
u_{1} \\
f
\end{array}\right] \in T_{\max }
$$

and the corresponding Weyl function is $m(\lambda)=m_{0} \lambda$.

The system $S[\widetilde{R}, \widetilde{W}]$ with $\widetilde{R}(x)=l_{1} H(x-1), \widetilde{W}(x)=m_{0} H(x), x \in[0,2]$ is equivalent to the system $S[R, W]$ in the sense that its Weyl function corresponding to the boundary triple (3.49) coincides with $m(\lambda)=m_{0} \lambda$ and the monodromy matrix $\widetilde{U}(2, \lambda)$ of this system coincides with $U\left(l_{1}, \lambda\right)$. The advantage of system $S[\widetilde{R}, \widetilde{W}]$ is that the elementary factors of $\widetilde{U}(2, \lambda)$ from its factorization

$$
\tilde{U}(2, \lambda)=U^{(1)}(\lambda) U^{(0)}(\lambda), \quad U^{(1)}(\lambda)=\left(\begin{array}{cc}
1 & l_{1} \\
0 & 1
\end{array}\right), \quad U^{(0)}(\lambda)=\left(\begin{array}{cc}
1 & 0 \\
-\lambda m_{0} & 1
\end{array}\right)
$$

can be also treated as monodromy matrices of systems $S[0, \widetilde{W}]$ on the interval $[0,1]$ and $S[\widetilde{R}, 0]$ on $[1,2]$, respectively. 


\section{Integral Systems in the Limit Point Case}

\subsection{The Strong Limit Point Condition}

The next lemma is an analog of a result in [16, Lemma] in the case of integral systems.

Lemma 4.1 Let $f$ be a (not necessarily strictly) monotone function on $\left[b_{0}, b\right)$ such that either $f(x) \rightarrow \pm \infty$ or $f(x) \rightarrow 0$ as $x \rightarrow b$ and let $f(x) \neq 0$ on $\left[b_{0}, b\right)$. Then

$$
\lim _{x \rightarrow b} \int_{b_{0}}^{x} d f / f= \pm \infty
$$

Proof We will prove the lemma in the case $f>0, f \rightarrow 0$. The proof in the other cases is similar. Let $D_{f}$ be the set of the points of discontinuity of $f$ on $\left[b_{0}, b\right)$. One can write

$$
\int_{\left[b_{0}, x\right)} \frac{d f}{f}=\int_{\left[b_{0}, x\right) \backslash D_{f}} \frac{d f}{f}+\int_{\left[b_{0}, x\right) \cap D_{f}} \frac{d f}{f} .
$$

Notice that both the integrals on the right hand side of (4.2) are negative, therefore if one of them diverges (as $x \rightarrow b$ ) then the assertion of the lemma holds.

Let $D_{f}=\left\{x_{n}\right\}_{n=0}^{\infty}$. Consider the following inequality

$$
\frac{f_{+}\left(x_{n}\right)-f_{-}\left(x_{n}\right)}{f\left(x_{n}\right)} \leq \frac{f_{+}\left(x_{n}\right)-f_{-}\left(x_{n}\right)}{f_{-}\left(x_{n}\right)}=\frac{f_{+}\left(x_{n}\right)}{f_{-}\left(x_{n}\right)}-1<0
$$

and the associated series

$$
\sum_{n=0}^{\infty}\left(\frac{f_{+}\left(x_{n}\right)}{f_{-}\left(x_{n}\right)}-1\right)
$$

If series (4.3) diverges then the following integral

$$
\int_{\left[b_{0}, b\right) \cap D_{f}} \frac{d f}{f}=\sum_{x_{n} \in D_{f}} \frac{f_{+}\left(x_{n}\right)-f_{-}\left(x_{n}\right)}{f\left(x_{n}\right)}
$$

diverges as well, so the assertion of the lemma holds immediately.

Assume now that series (4.3) converges and denote $a_{n}:=1-f_{+}\left(x_{n}\right) / f_{-}\left(x_{n}\right)$. Notice that the measure $d \log (f)$ is absolutely continuous with respect to $d f$ and therefore there exists the Radon-Nikodym derivative $d \log (f) / d f \in L^{1}(d f)$ which has the representation (see $[6,5.3$, formula (3.5)])

$$
\frac{d \log (f)}{d f}= \begin{cases}1 / f(x), & x \in\left[b_{0}, b\right) \backslash D_{f}, \\ \left(\log f_{+}(x)-\log f_{-}(x)\right) /\left(f_{+}(x)-f_{-}(x)\right), & x \in D_{f} .\end{cases}
$$


Now we get by the Radon-Nikodym theorem

$$
\log \frac{f_{-}(x)}{f_{+}\left(b_{0}\right)}=\int_{\left[b_{0}, x\right)} \frac{d \log (f)}{d f} d f=\int_{\left[b_{0}, x\right) \backslash D_{f}} \frac{d f}{f}+\int_{D_{f}} \frac{\log f_{+}(x)-\log f_{-}(x)}{f_{+}(x)-f_{-}(x)} d f
$$

and hence

$$
\int_{\left[b_{0}, x\right) \backslash D_{f}} \frac{d f}{f}=\log \frac{f_{-}(x)}{f_{+}\left(b_{0}\right)}+\sum_{x_{n} \in D_{f}} \log \frac{f_{-}\left(x_{n}\right)}{f_{+}\left(x_{n}\right)} .
$$

One can see from the following inequality

$$
0<\log \frac{f_{-}\left(x_{n}\right)}{f_{+}\left(x_{n}\right)} \leq \frac{f_{-}\left(x_{n}\right)-f_{+}\left(x_{n}\right)}{f_{+}\left(x_{n}\right)}=\frac{a_{n}}{1-a_{n}}
$$

that the series

$$
\sum_{n=1}^{\infty} \log \frac{f_{-}\left(x_{n}\right)}{f_{+}\left(x_{n}\right)}
$$

converges provided the series $\sum_{1}^{\infty} a_{n}$ converges. Therefore, the integral on the left hand side of (4.5) diverges which completes the proof.

Definition 4.2 ([14-16]) Let the system $S[R, W]$ be singular at $b$. It is said to be in the strong limit point case if

$$
\lim _{x \rightarrow b} u_{1}(x) v_{2}(x)=0 \text { for any } \quad\left(u_{1}, u_{2}, f\right),\left(v_{1}, v_{2}, g\right) \in \mathcal{T}
$$

and it is said to have the Dirichlet property if

$$
\int_{0}^{b}\left|u_{2}(t)\right|^{2} d R(t)<\infty \quad \text { for any } \quad\left(u_{1}, u_{2}, f\right) \in \mathcal{T}
$$

Theorem 4.3 Let the system $S[R, W]$ be singular at $b$. Then the following statements are equivalent:

(LP) The system $S[R, W]$ is in the limit point case.

(D) The system $S[R, W]$ has the Dirichlet property.

$\left(S L P^{*}\right)$ For any $\left(u_{1}, u_{2}, f\right) \in \mathcal{T}$ the following equality holds

$$
\lim _{x \rightarrow b} u_{1}(x) u_{2}(x)=0
$$

(SLP) The system $S[R, W]$ is in the strong limit point case. 
Proof Without loss of generality we assume here that the functions $u_{1}, u_{2}$, and $f$ are real-valued. By the first Green's identity (2.26) one obtains

$$
\int_{0}^{x} u_{2}^{2} d R=\int_{0}^{x} f u_{1} d W+\left.u_{1} u_{2}\right|_{0} ^{x}
$$

and hence

$$
\lim _{x \rightarrow b} u_{1}(x) u_{2}(x)=d,
$$

where $d \in \mathbb{R}$ if the Dirichlet property holds and $d=+\infty$ otherwise.

Let us start with the implication (LP) $\Rightarrow(\mathrm{D})$. For this purpose we assume the contrary i.e. the system $S[R, W]$ is in the limit point case but $d=+\infty$. Notice, that according to Assumption 2.8 the functions $R$ and $W$ do not have common points of discontinuity, therefore neither do the functions $u_{1}$ and $u_{2}$. It implies that both $u_{1}$ and $u_{2}$ preserve their signs on some interval $\left[b_{0}, b\right)$ (otherwise they would have to share a jump from a positive to a negative value or vice versa). It follows from (2.17) that the function $u_{1}$ is either positive and increasing or negative and decreasing. If $1 \notin \mathcal{L}^{2}(d W)$ then it immediately results as $u_{1} \notin \mathcal{L}^{2}(d W)$.

In the case if $1 \in \mathcal{L}^{2}(d W)$ (and hence $R \notin \mathcal{L}^{2}(d W)$ ) the implication $f \in$ $\mathcal{L}^{2}(d W) \Rightarrow f \in \mathcal{L}^{1}(W)$ is valid and hence (see (3.15)) there exists a finite limit $u_{2}(b):=\lim _{x \rightarrow b} u_{2}(x)$. The limit $u_{2}(b)$ must be zero, otherwise from

$$
\left|u_{1}(x)-u_{1}\left(b_{0}\right)\right|=\left|\int_{b_{0}}^{x} u_{2} d R\right| \geq \frac{\left|u_{2}(b)\right|}{2}\left(R(x)-R\left(b_{0}\right)\right)
$$

one gets $u_{1} \notin \mathcal{L}^{2}(d W)$. One can see that $1 / u_{2} \notin \mathcal{L}^{2}(d W)$. Indeed, if $1 / u_{2} \in \mathcal{L}^{2}(d W)$ then the integral

$$
\int_{0}^{x} \frac{f}{u_{2}} d W=-\int_{0}^{x} \frac{d u_{2}}{u_{2}}
$$

converges as $x \rightarrow b$, which contradicts to Lemma 4.1. Since $d=+\infty$, the estimate $\left|u_{1}\right|>1 /\left|u_{2}\right|$ hold on some interval $\left[b_{0}, b\right)$ and provides again $u_{1} \notin \mathcal{L}^{2}(d W)$. This completes the proof of the implication (LP) $\Rightarrow(\mathrm{D})$.

Now let us prove the implication (D) $\Rightarrow\left(\mathrm{SLP}^{*}\right)$. We first will show that $d=0$. In the case $1 \in \mathcal{L}^{2}(d W)$ the reasoning of the previous paragraph can be used to show that $u_{1} \notin \mathcal{L}^{2}(d W)$ for every non-zero $d$. In the case $1 \notin \mathcal{L}^{2}(d W)$ the reasoning above shows again that $u_{1} \notin \mathcal{L}^{2}(d W)$ for every $d>0$. Therefore we assume $d<0$ and get that $u_{1}$ is either positive and decreasing or negative and increasing on some interval $\left[b_{0}, b\right)$, namely $u_{1} \rightarrow 0$ as $x \rightarrow b$. From $\left|u_{1} u_{2}\right|>|d| / 2$ on $\left[b_{0}, b\right)$ (with a possible change of point $b_{0}$ ) we obtain the following inequality

$$
\int_{b_{0}}^{b} u_{2}^{2} d R=\int_{b_{0}}^{b} u_{2} d f_{1}>\frac{d}{2} \int_{b_{0}}^{b} \frac{d u_{1}}{u_{1}}=+\infty
$$


The left hand side converges by the assumption (D) but the right hand side diverges due to Lemma 4.1. This contradiction proves that $d=0$. Thus, implication (D) $\Rightarrow$ (SLP) is valid.

As is known (see [41, Theorem 4.3]), the system $S[R, W]$ is in the limit point case if and only if for every $\left(u_{1}, u_{2}, f\right)$ and $\left(v_{1}, v_{2}, g\right)$ from $\mathcal{T}$

$$
\lim _{x \rightarrow b}[u, v]_{x}=\lim _{x \rightarrow b}\left(u_{1}(x) v_{2}(x)-u_{2}(x) v_{1}(x)\right)=0 .
$$

In order to prove the implication (SLP*) $\Rightarrow($ SLP) we notice first that by Lemma 3.3 the system $S[R, W]$ cannot be in the limit circle case since (4.8) holds for every $\left(u_{1}, u_{2}, f\right) \in \mathcal{T}$. The condition (4.6) follows from (4.8), (4.9) and the following equality (cf. [16])

$$
2 u_{1}(x) v_{2}(x)=\left(u_{1}+v_{1}\right)\left(u_{2}+v_{2}\right)+[u, v]_{x}=0 .
$$

Assume that the statement (SLP) holds, i.e. condition (4.6) is satisfied for every $\left(u_{1}, u_{2}, f\right)$ and $\left(v_{1}, v_{2}, g\right)$ from $\mathcal{T}$. Then, clearly, (4.9) holds for every $\left(u_{1}, u_{2}, f\right)$ and $\left(v_{1}, v_{2}, g\right)$ from $\mathcal{T}$ and hence the system $S[R, W]$ is in the limit point case. This proves the implication (SLP) $\Rightarrow$ (LP).

Remark 4.4 In the case of absolutely continuous $R$ and $W$ the implication (LP) $\Rightarrow$ (SLP) for the system $S[R, W]$ was proved in [28], see also [16].

\subsection{Boundary Triples for Integral Systems in the Limit Point Case}

Definition 4.5 Let the system $S[R, W]$ be in the limit point case at $b$. Then for each $\lambda \in \mathbb{C} \backslash \mathbb{R}$ there is a unique coefficient $m_{N}(\lambda)$, such that

$$
\psi_{1}(\cdot, \lambda)=s_{1}(\cdot, \lambda)-m_{N}(\lambda) c_{1}(\cdot, \lambda) \in \mathcal{L}^{2}(d W)
$$

The function $m_{N}$ is called the Neumann $m$-function of the system (1.1) on $I$ and the function $\psi(t, \lambda)$ is called the Weyl solution of the system $S[R, W]$ on $I$.

Let us collect some statements concerning boundary triples for $S^{*}$, which were partially formulated in $[40,41]$.

Proposition 4.6 Let the system $S[R, W]$ be in the limit point case at $b$, and let $T=$ $T_{\min }$. Then:

(i) $T$ is a symmetric nonnegative operator in $L^{2}(d W)$ with deficiency indices $(1,1)$.

(ii) The triple $\Pi=\left(\mathbb{C}, \Gamma_{0}, \Gamma_{1}\right)$, where

$$
\Gamma_{0} \boldsymbol{u}=u_{2}(0), \quad \Gamma_{1} \boldsymbol{u}=-u_{1}(0), \quad \boldsymbol{u} \in T^{*},
$$

is a boundary triple for $T^{*}$. 
(iii) The defect subspace $\mathfrak{N}_{\lambda}$ is spanned by the Weyl solution $\psi_{1}(t, \lambda)$, and the Weyl function $m(\lambda)$ of $T$ corresponding to the boundary triple $\Pi$ coincides with the Neumann m-function of the system $S[R, W]$ on $I$ :

$$
m(\lambda)=-\frac{\psi_{1}(0, \lambda)}{\psi_{2}(0, \lambda)}=m_{N}(\lambda)
$$

(iv) The Weyl function $m(\lambda)$ of $T$ corresponding to the boundary triple $\Pi$ coincides with the principal Titchmarsh-Weyl coefficient $q(\lambda)$ of the system $S[R, W]$ on I and belongs to the Stieltjes class $\mathcal{S}$.

(v) If $W(b)<\infty$ then the Weyl function $m_{N}$ of $T_{N}$ admits the representation

$$
m_{N}(\lambda)=-\frac{1}{W(b) \cdot \lambda}+\tilde{m}(\lambda)
$$

where $\tilde{m}$ is a function from $\mathcal{S}$ such that $\lim _{y \downarrow}$ y $\tilde{m}(i y)=0$.

Proof 1. At first we show (i)-(ii). Since (1.1) is in the limit point case at $b$,

$$
\lim _{x \rightarrow b}[u, \bar{v}]_{x}=0 \text { for } \boldsymbol{u}=\left[\begin{array}{c}
\pi u_{1} \\
\pi f
\end{array}\right], \boldsymbol{v}=\left[\begin{array}{c}
\pi v_{1} \\
\pi g
\end{array}\right] \in T_{\max }
$$

and hence the generalized Green's identity (2.27) is of the form

$$
\int_{0}^{b}\left(f \overline{v_{1}}-u_{1} \bar{g}\right) d W(t)=-[u, \bar{v}]_{0}=u_{2}(0) \overline{v_{1}(0)}-u_{1}(0) \overline{v_{2}(0)}
$$

Therefore, the triple $\Pi$ in (4.11) is a boundary triple for $T^{*}$.

It follows from the first Green's identity (2.26) and Lemma 3.3 that for every $\boldsymbol{u} \in T$ the identity (3.31) holds and thus the linear relation $T$ is nonnegative.

2. Now (iii) is shown. In the limit point case there is only one linearly independent solution $\psi(\cdot, \lambda)$ of the system $S[R, W]$ such that $\psi_{1}(\cdot, \lambda) \in L^{2}(d W)$, see (4.10), and hence the defect subspace $\mathfrak{N}_{\lambda}:=\mathfrak{N}_{\lambda}\left(T^{*}\right)$ is spanned by the function $\psi_{1}(\cdot, \lambda)$. Denote $\boldsymbol{u}(t, \lambda)=\left(\psi_{1}(\cdot, \lambda), \lambda \psi_{1}(\cdot, \lambda)\right)^{T} \in \widehat{\mathfrak{N}}_{\lambda}\left(T^{*}\right)$. It follows from (4.11) that

$$
\Gamma_{0} \boldsymbol{u}(\cdot, \lambda)=\psi_{2}(0, \lambda)=1, \quad \Gamma_{1} \boldsymbol{u}(\cdot, \lambda)=-\psi_{1}(0, \lambda)=m_{N}(\lambda)
$$

This yields formula (4.12).

3. Now we show (iv). If $\lambda \in \mathbb{R}_{\text {- }}$ then it follows from Lemma 3.1 that the function $\frac{s_{1}(x, \lambda)}{c_{1}(x, \lambda)}$ is increasing and bounded from above. Therefore, the following limit

$$
q(\lambda):=\lim _{x \rightarrow b} \frac{s_{1}(x, \lambda)}{c_{1}(x, \lambda)}
$$

exists and is nonnegative for every $\lambda \in \mathbb{R}_{-}$. By Stieltjes-Vitaly theorem the function $q$ is holomorphic on $\mathbb{C} \backslash[0, \infty)$. The function $q$ belongs to the Stieltjes class $\mathcal{S}$, since it is nonnegative for every $\lambda \in \mathbb{R}_{-}$. Since $\frac{s_{1}(x, \lambda)}{c_{1}(x, \lambda)}$ belongs to the Weyl disc $D_{x}(\lambda)$ and 
the system $S[R, W]$ is limit point at $b$, for every $\lambda \in \mathbb{C}_{+} \cup \mathbb{C}_{-}$the following equality holds

$$
q(\lambda)=\lim _{x \rightarrow b} \frac{s_{1}(x, \lambda)}{c_{1}(x, \lambda)}=m_{N}(\lambda) .
$$

4. Assume that $W(b)<+\infty$. Let us consider the family of von Neumann $m$ functions $m_{N}^{x}(\lambda)=\frac{s_{2}(x, \lambda)}{c_{2}(x, \lambda)}$ converging to $m_{N}(\lambda)$ as $x \rightarrow b-$. Due to equality (3.12)

$$
\frac{1}{m_{N}^{x}(\lambda)}=\frac{c_{2}(x, \lambda)}{s_{2}(x, \lambda)}=\int_{0}^{x} \frac{-\lambda}{s_{2}(x, \lambda) s_{2+}(x, \lambda)} d W(x)
$$

Since $s_{2}(x, \lambda) \geq 1$ for $x \in[0, b)$ and $\lambda \in \mathbb{R}_{-}$there exists the limit

$$
\frac{-1}{\lambda m_{N}(\lambda)}=\lim _{x \rightarrow b} \frac{-c_{2}(x, \lambda)}{\lambda s_{2}(x, \lambda)}=\int_{0}^{b} \frac{1}{s_{2}(x, \lambda) s_{2+}(x, \lambda)} d W(x) .
$$

Due to Lemma 3.1

$$
\lim _{\lambda \downarrow 0} \frac{1}{s_{2}(x, \lambda) s_{2+}(x, \lambda)}=1, \quad \text { and } \quad\left|\frac{1}{s_{2}(x, \lambda) s_{2+}(x, \lambda)}\right| \leq 1 \quad \text { for } \quad x \in[a, b) .
$$

Hence one obtains by the Lebesgue bounded convergence Theorem

$$
\lim _{\lambda \rightarrow 0} \frac{1}{-\lambda m_{N}(\lambda)}=\int_{[0, b)} d W=W(b) .
$$

This implies (v).

\subsection{The Canonical Singular Continuation of a Regular Integral System}

If the integral system $S[R, W]$ is regular at $b$ then due to Remark 3.9 we can assume without loss of generality that $b<\infty$.

Definition 4.7 For a regular system $S[R, W]$ with $b<\infty$ we define the extended functions

$$
\widetilde{R}(x):=\left\{\begin{array}{l}
R(x): x \in[0, b], \\
R(b): x \in(b, \infty),
\end{array} \quad \widetilde{W}(x):=\left\{\begin{array}{cc}
W(x): & x \in[0, b], \\
W(b)+x-b: x \in(b, \infty) .
\end{array}\right.\right.
$$

The integral system $S[\widetilde{R}, \widetilde{W}]$ corresponding to

$$
\widetilde{u}(x, \lambda)=\widetilde{u}(0, \lambda)+\int_{0}^{x}\left[\begin{array}{cc}
0 & d \widetilde{R}(t) \\
-\lambda d \widetilde{W}(t) & 0
\end{array}\right] \widetilde{u}(t, \lambda), \quad x \in[0, \infty)
$$

will be called the canonical singular continuation of a regular integral system $S[R, W]$. 
Proposition 4.8 Let the integral system $S[R, W]$, see (1.1), be regular at $b<\infty$. Then the principal Titchmarsh-Weyl coefficient $\widetilde{q}$ of its canonical singular continuation $S[\widetilde{R}, \widetilde{W}]$ coincides with the principal Titchmarsh-Weyl coefficient $q$ of the system $S[R, W]$ :

$$
\widetilde{q}(\lambda)=q(\lambda), \quad \lambda \in \mathbb{C} \backslash \mathbb{R} .
$$

Proof Let the pair $u_{1}, u_{2}$ satisfy the integral system $S[R, W]$ for some $\lambda \in \mathbb{C} \backslash \mathbb{R}$ and let $\tilde{u}_{1}, \tilde{u}_{2}$ be the continuations of $u_{1}, u_{2}$ to the interval $[0,+\infty)$ given by

$$
\left\{\begin{array}{l}
\tilde{u}_{1}(x, \lambda)=u_{1}(b, \lambda), \quad x \in(b, \infty), \\
\tilde{u}_{2}(x, \lambda)=u_{2}(b, \lambda)-\lambda u_{1}(b, \lambda)(x-b), \quad x \in(b, \infty) .
\end{array}\right.
$$

Then the pair $\tilde{u}_{1}, \tilde{u}_{2}$ satisfies the integral system (4.19). If $c_{1}, c_{2}$ and $s_{1}, s_{2}$ are solutions of (1.1) according to the initial conditions (1.7) then the continuations $\widetilde{c}_{1}, \widetilde{c}_{2}$ and $\widetilde{s}_{1}, \widetilde{s}_{2}$ are solutions of the integral system (4.19) with the same initial conditions (1.7).

In view of (4.21) the principal Titchmarsh-Weyl coefficient $\widetilde{q}$ of the canonical singular continuation $S[\widetilde{R}, \widetilde{W}]$ is of the form

$$
\widetilde{q}(\lambda)=\lim _{x \rightarrow \infty} \frac{\widetilde{s}_{1}(x, \lambda)}{\widetilde{c}_{1}(x, \lambda)}=\lim _{x \rightarrow \infty} \frac{s_{1}(x, \lambda)}{c_{1}(x, \lambda)}=q(\lambda) .
$$

\section{Dual Integral Systems}

Definition 5.1 The dual system $\widehat{S}[R, W]$ to a singular system $S[R, W]$ is defined by changing the roles of $R$ and $W$ in (1.1), that is $\widehat{S}[R, W]=S[W, R]$ and

$$
\widehat{u}(x, \lambda)=\widehat{u}(0, \lambda)+\int_{0}^{x}\left[\begin{array}{cc}
0 & d W(t) \\
-\lambda d R(t) & 0
\end{array}\right] \widehat{u}(t, \lambda), \quad x \in[0, b) .
$$

In case the system $S[R, W]$ is regular, we will denote by $\widehat{S}[R, W]$ the dual to its canonical singular continuation: $\widehat{S}[R, W]=S[\widetilde{W}, \widetilde{R}]$.

Let $\widehat{s}(\cdot, \lambda)$ and $\widehat{c}(\cdot, \lambda)$ be the unique solutions of $(5.1)$ satisfying the initial conditions

$$
\widehat{c}_{1}(0, \lambda)=1, \widehat{c}_{2}(0, \lambda)=0, \quad \text { and } \widehat{s}_{1}(0, \lambda)=0, \widehat{s}_{2}(0, \lambda)=1 \text {. }
$$

Theorem 5.2 Let $U(x, \lambda)$ and $\widehat{U}(x, \lambda)$ be the fundamental matrices of the system $S[R, W]$ and its dual system $\widehat{S}[R, W]$ respectively. Let $m_{N}$ and $\widehat{m}_{N}$ be the Neumann $m$-functions of the systems $S[R, W]$ and $\widehat{S}[R, W]$ in the sense of Definitions 3.5, 4.5. Then:

(i) The matrices $U(x, \lambda)$ and $\widehat{U}(x, \lambda)$ are related by

$$
\widehat{U}(x, \lambda)=D(\lambda)^{-1} U(x, \lambda) D(\lambda), \text { where } D(\lambda)=\left(\begin{array}{cc}
0 & -\lambda^{-1} \\
1 & 0
\end{array}\right) .
$$


(ii) If the system $S[R, W]$ is singular at $b$, then

$$
\widehat{m}_{N}(\lambda)=-\frac{1}{\lambda m_{N}(\lambda)}
$$

(iii) If $S[R, W]$ is regular at $b$, then

$$
\widehat{m}_{N}(\lambda)=\frac{\widehat{s}_{2}(b, \lambda)}{\widehat{c}_{2}(b, \lambda)}=-\frac{c_{1}(b, \lambda)}{\lambda s_{1}(b, \lambda)}=-\frac{1}{\lambda m_{N D}(\lambda)},
$$

where $m_{N D}(\lambda)$ is the Neumann $m$-function of system $S[R, W]$, subject to the boundary condition $u_{1}(b)=0$, see Definition 3.7.

(iv) The principal Titchmarsh-Weyl coefficients $q$ and $\widehat{q}$ of $S[R, W]$ and $\widehat{S}[R, W]$ are connected by the equality

$$
\widehat{q}(\lambda)=-\frac{1}{\lambda q(\lambda)}, \quad \lambda \in \mathbb{C} \backslash \mathbb{R}_{+} .
$$

Proof 1. At first (i) is shown. A straightforward calculation shows that the solutions $\widehat{s}(\cdot, \lambda)$ and $\widehat{c}(\cdot, \lambda)$ of $(5.1)$ are related to the solutions $s(\cdot, \lambda)$ and $c(\cdot, \lambda)$ of $(1.1)$ by the equalities

$$
\left[\begin{array}{c}
\widehat{c}_{1}(\cdot, \lambda) \\
\widehat{c}_{2}(\cdot, \lambda)
\end{array}\right]=\left[\begin{array}{c}
s_{2}(\cdot, \lambda) \\
-\lambda s_{1}(\cdot, \lambda)
\end{array}\right], \quad\left[\begin{array}{c}
\widehat{s}_{1}(\cdot, \lambda) \\
\widehat{s}_{2}(\cdot, \lambda)
\end{array}\right]=\left[\begin{array}{c}
-\lambda^{-1} c_{2}(\cdot, \lambda) \\
c_{1}(\cdot, \lambda)
\end{array}\right]
$$

The equality (5.3) follows from (5.7) and (3.1).

System $S[R, W]$ is regular at $b$ if and only if both $S[R, W]$ and $\widehat{S}[R, W]$ are in the limit circle case at $b$. Therefore the proof of (ii) can be splitted into the following three cases 2-4.

2. Both $S[R, W]$ and $\widehat{S}[R, W]$ are in the limit point case at $b$ :

Let $m_{N}$ be the Neumann $m$-function of the systems $S[R, W]$, see Definition 4.5 , and let $\psi_{1}(\cdot, \lambda)$ be the corresponding Weyl solution of the system $S[R, W]$. Then the vector function

$$
\widehat{\psi}(\cdot, \lambda):=\left[\begin{array}{c}
\widehat{s}_{1}(\cdot, \lambda) \\
\widehat{s}_{2}(\cdot, \lambda)
\end{array}\right]+\frac{1}{\lambda m_{N}(\lambda)}\left[\begin{array}{c}
\widehat{c}_{1}(\cdot, \lambda) \\
\widehat{c}_{2}(\cdot, \lambda)
\end{array}\right]=\left[\begin{array}{c}
-\frac{1}{\lambda} c_{2}(\cdot, \lambda)+\frac{1}{\lambda m_{N}(\lambda)} s_{2}(\cdot, \lambda) \\
c_{1}(\cdot, \lambda)-\frac{1}{m_{N}(\lambda)} s_{1}(\cdot, \lambda)
\end{array}\right]
$$

is a solution of the system (5.1). Moreover, due to Lemma $4.3 \widehat{\psi}_{1}(\cdot, \lambda)=$ $\frac{1}{\lambda m_{N}(\lambda)} \psi_{2}(\cdot, \lambda)$ belongs to $\mathcal{L}^{2}(R)$. Therefore, $\widehat{\psi}_{1}(\cdot, \lambda)$ is the Weyl solution of the system $\widehat{S}[R, W]$ and the function $-\frac{1}{\lambda m_{N}(\lambda)}$ is the Neumann $m$-function of the system $\widehat{S}[R, W]$.

3. $S[R, W]$ is in the limit circle case and $\widehat{S}[R, W]$ is in the limit point case at $b$ : Let the function $\psi^{N}$ be defined by (3.24). Since (1.1) is in the limit circle case it follows from Lemma 3.3 that $\psi_{2}^{N} \in \mathcal{L}^{2}(R)$. Hence, $\widehat{\psi}(\cdot, \lambda)$ is a solution of the system $\widehat{S}[R, W]$, such that $\widehat{\psi}_{1}(\cdot, \lambda)=\frac{1}{\lambda m_{N}(\lambda)} \psi_{2}^{N}(\cdot, \lambda) \in \mathcal{L}^{2}(R)$. Therefore, $\widehat{\psi}_{1}$ is the Weyl 
solution of the system $\widehat{S}[R, W]$ and the function $-\frac{1}{\lambda m_{N}(\lambda)}$ is the Neumann $m$-function of the systems $\widehat{S}[R, W]$.

4. $S[R, W]$ is in the limit point case and $\widehat{S}[R, W]$ is in the limit circle case at $b$ : As was shown on Step 3 the Neumann $m$-function $\widehat{m}_{N}(\lambda)$ of the systems $\widehat{S}[R, W]$ subject to the boundary condition $\widehat{\psi}_{2}(b, \lambda)=0$ is connected with the Neumann $m$ function $m_{N}(\lambda)$ of the system $S[R, W]$ by the equality

$$
m_{N}(\lambda)=-\frac{1}{\lambda \widehat{m}_{N}(\lambda)}
$$

which is equivalent to (5.4).

5. Now (iii) is shown. Let $m_{N D}(\lambda)$ be the Neumann $m$-function of the system $S[R, W]$, subject to the boundary condition $(3.42)$ and let $\psi_{1}^{N D}(\cdot, \lambda)$ be the corresponding Weyl solution of the system $S[R, W]$ defined by (3.41). By definition $\psi_{1}^{N D}(b, \lambda)=0$. Then the vector function

$$
\begin{aligned}
\widehat{\psi}(\cdot, \lambda) & :=\left[\begin{array}{c}
\widehat{s}_{1}(\cdot, \lambda) \\
\widehat{s}_{2}(\cdot, \lambda)
\end{array}\right]+\frac{1}{\lambda m_{N D}(\lambda)}\left[\begin{array}{c}
\widehat{c}_{1}(\cdot, \lambda) \\
\widehat{c}_{2}(\cdot, \lambda)
\end{array}\right] \\
& =-\frac{1}{m_{N D}(\lambda)}\left[\begin{array}{c}
-\frac{1}{\lambda}\left(s_{2}(\cdot, \lambda)-m_{N D}(\lambda) c_{2}(\cdot, \lambda)\right) \\
s_{1}(\cdot, \lambda)-m_{N D}(\lambda) c_{1}(\cdot, \lambda)
\end{array}\right]
\end{aligned}
$$

is a solution of the system (5.1) such that $\widehat{\psi}_{2}(b, \lambda)=\psi_{1}^{N D}(b, \lambda)=0$. Therefore, the function $\frac{-1}{\lambda m_{N D}(\lambda)}$ is the Neumann $m$-function of the systems $\widehat{S}[R, W]$, subject to the boundary condition $\widehat{\psi}_{2}(b, \lambda)=0$.

6. Finally (iv) is shown. If the integral system $S[R, W]$ is singular at $b$ then the Neumann $m$-function $m_{N}$ (resp. $\widehat{m}_{N}$ ) coincides with the principal Titchmarsh-Weyl coefficient $q$ of the system $S[R, W]$ (resp. $\widehat{q}$ of the system $\widehat{S}[R, W]$ ), see Propositions 3.6, 4.6. Therefore, (5.6) is implied by (5.4).

If the system $S[R, W]$ is regular at $b$ then by Propositions $4.8 q$ coincides with the principal Titchmarsh-Weyl coefficient $\widetilde{q}$ of the canonical singular continuation $S[\widetilde{R}, \widetilde{W}]$ of the system $S[R, W]$ to $[0,+\infty)$, see (4.18). By the statement of the above paragraph the principal Titchmarsh-Weyl coefficient $\widehat{q}$ of the dual system $S[\widetilde{W}, \widetilde{R}]$ is of the form

$$
\widehat{q}(\lambda)=-\frac{1}{\lambda \widetilde{q}(\lambda)}=-\frac{1}{\lambda q(\lambda)},
$$

and (5.6) is shown.

Since the relation of duality for integral systems is reflexive one derives from the proof of Theorem 5.2 the following statement.

Corollary 5.3 Let the system $S[R, W]$ be in the limit point case and let $\widehat{S}[R, W]$ be in the limit circle case at $b$. Let $\psi_{1}(\cdot, \lambda)$ be the corresponding Weyl solution of the system $S[R, W]$. Then

$$
\lim _{x \rightarrow b} \psi_{1}(x, \lambda)=0 .
$$


Proof As it was mentioned in the proof of Theorem 5.2 (Step 3), the Weyl solution $\psi(\cdot, \lambda)$ of the system $S[R, W]$ is connected with the Weyl solution $\widehat{\psi}^{N}(\cdot, \lambda)$ of the dual system $(1.1)$ by the equality $\psi_{1}(\cdot, \lambda)=\frac{1}{\lambda \widehat{m}_{N}(\lambda)} \widehat{\psi}_{2}^{N}(\cdot, \lambda)$. Since $\widehat{\psi}_{2}^{N}(b, \lambda)=0$ one obtains (5.8).

Remark 5.4 Formula (5.4) was proved in [29] for Krein strings and in [33] for integral systems. However, in [33] it was overlooked that formula (5.4) fails to hold in the regular case and should be replaced by (5.5).

\section{The Connection Between Integral and Canonical Systems}

Let $H$ be a real, symmetric and non-negative locally integrable $2 \times 2$-matrix function on the interval $\left[0, l_{H}\right)$ for some $l_{H} \in(0, \infty]$. In this section we consider initial value problems of the form

$$
J y^{\prime}(x)=-z H(x) y(x), x \in\left[0, l_{H}\right), \quad y_{1}(0)=0, \quad J=\left[\begin{array}{cc}
0 & -1 \\
1 & 0
\end{array}\right],
$$

with $y(x)=\left(y_{1}(x) y_{2}(x)\right)^{T}$ and a complex parameter $z$. Here the differential equation in $(6.1)$ is considered to hold almost everywhere on $\left[0, l_{H}\right)$. The fundamental matrix function

$$
W(x, z)=\left[\begin{array}{ll}
w_{11}(x, z) & w_{12}(x, z) \\
w_{21}(x, z) & w_{22}(x, z)
\end{array}\right]
$$

of a canonical system (6.1) with Hamiltonian $H$ is defined as the transpose of the fundamental solution of (6.1), i.e. solution of the integral equation

$$
W(x, z) J-J=z \int_{0}^{x} W(s, z) H(s) d s .
$$

This corresponds to the notation used in [34].

Note that $W(0, z)=I$. At $l_{H}$ for the canonical system (6.1) Weyl's limit point case prevails if and only if

$$
\int_{0}^{l_{H}} \operatorname{trace} H(x) d x=\infty,
$$

and from now on we assume that for each Hamiltonian $H$ the relation (6.3) holds, and that $H$ is not identically equal to diag $\left(\begin{array}{ll}1 & 0\end{array}\right)$ on the interval $[0, \infty)$. Then the limit point case prevails at $l_{H}$, and it follows that for each $\omega \in \tilde{\mathcal{N}}:=\mathcal{N} \cup\{\infty\}$ and $z \in \mathbb{C}^{+}$ the limit

$$
Q(z):=\lim _{x \rightarrow l_{H}} \frac{w_{11}(x, z) \omega(z)+w_{12}(x, z)}{w_{21}(x, z) \omega(z)+w_{22}(x, z)}
$$

exists, is independent of $\omega$, and, as a function of $z$, belongs to the set of HerglotzNevanlinna functions $\mathcal{N}$ (see, e.g., [7]). The function $Q$ is called the Titchmarsh-Weyl coefficient of the canonical system (6.1) or of the Hamiltonian $H$. 
The following intervals play a special role in the sequel (see $[9,24])$. Let $\xi_{\phi}:=$ $(\cos \phi, \sin \phi)^{T}$ for some $\phi \in[0, \pi)$. The open interval $I_{\phi} \subset\left[0, l_{H}\right)$ is called $H$ indivisible of type $\phi$ if the relation

$$
\xi_{\phi}^{T} J H=0 \text {, a.e. on } I_{\phi},
$$

holds. In particular, det $H=0$ a.e. on $I_{\phi}$. An $H$-indivisible interval is called maximal if it is not a proper subset of another $H$-indivisible interval.

A Hamiltonian $H$ is called trace normed if trace $H(x)=1$ a.e. on $[0, \infty)$. For the class of trace normed Hamiltonians a basic inverse result in [8] can be formulated as follows (see [42]): Each function $Q \in \mathcal{N}$ is the Titchmarsh-Weyl coefficient of a canonical system with a trace normed Hamiltonian $H$ on $[0, \infty)$ which is not equal to $\operatorname{diag}(1,0)$ a. e. on $[0, \infty)$; this correspondence is bijective if two Hamiltonians which coincide almost everywhere are identified.

In this section we associate with the integral system $S[R, W]$ a canonical system with diagonal Hamiltonian such that its Titchmarsh-Weyl coefficient $Q_{d}$ is related to the principal Titchmarsh-Weyl coefficient $q$ of $S[R, W]$ via the formula

$$
Q_{d}(z)=z q\left(z^{2}\right)
$$

Assume that the integral system $S[R, W]$ is singular at $d$, i.e.

$$
R(d)+W(d)=\infty .
$$

Let us set $x(t)=R(t)+W(t)$. Denote by $\mathcal{D}^{(1)}$ the set of points of discontinuity of $R$ and by $\mathcal{D}^{(2)}$ the set of points of discontinuity of $W$. Recall that by assumption $\mathcal{D}^{(1)} \cap \mathcal{D}^{(2)}=\emptyset$. Let $I_{x}$ be the range of the function $x(t)$. Then $I_{x}$ is a union of at most countable set of semi-intervals $(\xi, \eta]$, and $\mathbb{R} \backslash I_{x}$ is a union of semi-intervals $(x(t), x(t+)]$, where either $t \in \mathcal{D}^{(1)}$ or $t \in \mathcal{D}^{(2)}$.

On every semi-interval $(\xi, \eta] \subset I_{x}$ define the Hamiltonian $H_{d}$ by

$$
H_{d}(x):=\left[\begin{array}{cc}
h_{1}(x) & 0 \\
0 & h_{2}(x)
\end{array}\right], \quad \text { where } \quad h_{1}(x):=\frac{d R}{d x}, \quad h_{2}(x):=\frac{d W}{d x} .
$$

On the semi-interval $(x(t), x(t+)]$ with $t \in \mathcal{D}^{(1)}$ define the Hamiltonian $H_{d}$ by

$$
H_{d}(x):=\left[\begin{array}{ll}
1 & 0 \\
0 & 0
\end{array}\right]
$$

and on the semi-interval $(x(t), x(t+)]$ with $t \in \mathcal{D}^{(2)}$ define the Hamiltonian $H_{d}$ by

$$
H_{d}(x):=\left[\begin{array}{ll}
0 & 0 \\
0 & 1
\end{array}\right]
$$

Then $H_{d}$ is a trace normed Hamiltonian, i.e.

$$
\text { trace } H_{d}(x) \equiv 1 \text { for all } x \in \mathbb{R}_{+} \text {. }
$$


Let us consider the canonical system

$$
J y^{\prime}(x)=-z H_{d}(x) y(x) .
$$

The fundamental matrix $W_{d}$ of the canonical system (6.12) is then according to (6.2) the solution of the initial value problem

$$
W_{d}^{\prime}(x, z) J=z W_{d}(x, z) H_{d}(x), \quad x \in \mathbb{R}_{+}, \quad W_{d}(0, z)=I .
$$

Theorem 6.1 Let q be the principal Titchmarsh-Weyl coefficient of some integral system $S[R, W]$ such that (6.7) holds, and let $Q_{d}$ denote the Titchmarsh-Weyl coefficient corresponding to the Hamiltonian $H_{d}$. Then

(i) the fundamental matrix of the canonical system (6.12) takes the form

$$
W_{d}(x(t), z)=\left[\begin{array}{cc}
s_{2}\left(t, z^{2}\right) & z s_{1}\left(t, z^{2}\right) \\
\frac{1}{z} c_{2}\left(t, z^{2}\right) & c_{1}\left(t, z^{2}\right)
\end{array}\right], \quad x(t) \in I_{x}
$$

(ii) the following relation holds:

$$
Q_{d}(z)=z q\left(z^{2}\right)
$$

Proof On every semi-interval $(\xi, \eta] \subset I_{x}$ one obtains from (1.1)

$$
\begin{array}{ll}
d s_{1}\left(t, z^{2}\right)=s_{2}\left(t, z^{2}\right) d R(t), & d c_{1}\left(t, z^{2}\right)=c_{2}\left(t, z^{2}\right) d R(t), \\
d s_{2}\left(t, z^{2}\right)=-z^{2} s_{1}\left(t, z^{2}\right) d W(t), & d c_{2}\left(t, z^{2}\right)=-z^{2} c_{1}\left(t, z^{2}\right) d W(t) .
\end{array}
$$

Then it follows from (6.14) and (6.8) that

$$
W_{d}^{\prime}(x, z)=\left[\begin{array}{cc}
\frac{d s_{2}\left(t, z^{2}\right)}{d x} & z \frac{d s_{1}\left(t, z^{2}\right)}{d x} \\
\frac{1}{z} \frac{d c_{2}\left(t, z^{2}\right)}{d x} & \frac{d c_{1}\left(t, z^{2}\right)}{d x}
\end{array}\right]=\left[\begin{array}{cc}
\frac{d s_{2}\left(t, z^{2}\right)}{d W(t)} h_{2}(x) & z \frac{d s_{1}\left(t, z^{2}\right)}{d R(t)} h_{1}(x) \\
\frac{1}{z} \frac{d c_{2}\left(t, z^{2}\right)}{d W(t)} h_{2}(x) & \frac{d c_{1}\left(t, z^{2}\right)}{d R(t)} h_{1}(x)
\end{array}\right],
$$

and hence in view of (6.16), (6.17)

$$
W_{d}^{\prime}(x, z) J=\left[\begin{array}{cc}
z s_{2}\left(x, z^{2}\right) h_{1}(x) & z^{2} s_{1}\left(x, z^{2}\right) h_{2}(x) \\
c_{2}\left(x, z^{2}\right) h_{1}(x) & z c_{1}\left(x, z^{2}\right) h_{2}(x)
\end{array}\right] .
$$

On the other hand by (6.14) and (6.8)

$$
W_{d}(x, z) H_{d}(x)=\left[\begin{array}{cc}
s_{2}\left(x, z^{2}\right) h_{1}(x) & z s_{1}\left(x, z^{2}\right) h_{2}(x) \\
\frac{1}{z} c_{2}\left(x, z^{2}\right) h_{1}(x) & c_{1}\left(x, z^{2}\right) h_{2}(x)
\end{array}\right] .
$$

This proves that $W_{d}(x, z)$ is the fundamental matrix of the canonical system (6.12) on $I_{x}$.

Now let $(x(t), x(t+)]$ be a semi-interval with $t \in \mathcal{D}^{(1)}$ or $t \in \mathcal{D}^{(2)}$. Note that then $(x(t), x(t+))$ is an $H$-indivisible interval of type 0 if $t \in \mathcal{D}^{(1)}$ and an $H$-indivisible 
interval of type $\pi / 2$ if $t \in \mathcal{D}^{(2)}$. The fundamental matrix for $s \in[x(t), x(t+))$ is then of the form

$$
W_{d}(s, z)=W_{d}(x(t), z)\left(I-z(s-x(t)) H_{d}(s) J\right),
$$

so it remains to show that

$$
W_{d}(x(t+), z)=W_{d}(x(t), z)\left(I-z(x(t+)-x(t)) H_{d}(s) J\right),
$$

or, equivalently (since $H_{d}(s) J H_{d}(s)=0$ ), that according to (6.2) in both cases the integral equation

$$
W_{d}(x(t+), z) J-W_{d}(x(t), z) J=z \int_{x(t)}^{x(t+)} W_{d}(s, z) H_{d}(s) d s
$$

holds. Let for $i \in\{1,2\}$

$$
\Delta s_{i}\left(t, z^{2}\right)=s_{i}\left(t+, z^{2}\right)-s_{i}\left(t, z^{2}\right), \quad \Delta c_{i}\left(t, z^{2}\right)=c_{i}\left(t+, z^{2}\right)-c_{i}\left(t, z^{2}\right) .
$$

Assume that $t \in \mathcal{D}^{(1)}$ with $l_{1}=x(t+)-x(t)=R(t+)-R(t)$. Then it follows from (6.19) with equation (1.1) that $s_{2}\left(s, z^{2}\right)$ and $c_{2}\left(s, z^{2}\right)$ are constant for $s \in(x(t), x(t+)]$ and

$$
\Delta s_{1}\left(t, z^{2}\right)=s_{2}\left(t, z^{2}\right) l_{1}, \quad \Delta c_{1}\left(t, z^{2}\right)=c_{2}\left(t, z^{2}\right) l_{1} .
$$

it follows that

$$
W_{d}(x(t+), z) J-W_{d}(x(t), z) J=\left[\begin{array}{cc}
z \Delta s_{1}\left(x, z^{2}\right) & 0 \\
\Delta c_{1}\left(x, z^{2}\right) & 0
\end{array}\right]=l_{1}\left[\begin{array}{cc}
z s_{2}\left(s, z^{2}\right) & 0 \\
c_{2}\left(s, z^{2}\right) & 0
\end{array}\right] .
$$

On the other hand, the relation

$$
W_{d}(s, z) H_{d}(s)=\left[\begin{array}{cc}
s_{2}\left(s, z^{2}\right) & 0 \\
\frac{1}{z} c_{2}\left(s, z^{2}\right) & 0
\end{array}\right]
$$

holds and therefore

$$
z \int_{x(t)}^{x(t+)} W_{d}(s, z) H_{d}(s) d s=l_{1}\left[\begin{array}{cc}
z s_{2}\left(s, z^{2}\right) & 0 \\
c_{2}\left(s, z^{2}\right) & 0
\end{array}\right]
$$

and so (6.18) is shown.

Assume now that $t \in \mathcal{D}^{(2)}$ with $l_{2}=x(t+)-x(t)=W(t+)-W(t)$. Then it follows from (6.19) with equation (1.1) that $s_{1}\left(s, z^{2}\right)$ and $c_{1}\left(s, z^{2}\right)$ are constant for $s \in[x(t), x(t+)]$ and

$$
\Delta s_{2}\left(t, z^{2}\right)=-z^{2} s_{1}\left(t, z^{2}\right) l_{2}, \quad \Delta c_{2}\left(t, z^{2}\right)=-z^{2} c_{1}\left(t, z^{2}\right) l_{2} .
$$


It follows that

$$
W_{d}(x(t+), z) J-W_{d}(x(t), z) J=\left[\begin{array}{cc}
0 & -\Delta s_{2}\left(x, z^{2}\right) \\
0 & -\frac{1}{z} \Delta c_{2}\left(x, z^{2}\right)
\end{array}\right]=l_{2} z\left[\begin{array}{cc}
0 & z s_{1}\left(s, z^{2}\right) \\
0 & c_{1}\left(s, z^{2}\right)
\end{array}\right] .
$$

On the other hand, the relation

$$
W_{d}(s, z) H_{d}(s)=\left[\begin{array}{cc}
0 & z s_{1}\left(s, z^{2}\right) \\
0 & c_{1}\left(s, z^{2}\right)
\end{array}\right]
$$

holds and therefore

$$
z \int_{x(t)}^{x(t+)} W_{d}(s, z) H_{d}(s) d s=l_{2} z\left[\begin{array}{cc}
0 & z s_{1}\left(s, z^{2}\right) \\
0 & c_{1}\left(s, z^{2}\right)
\end{array}\right]
$$

holds, and so (6.18) is shown in that case.

The relation (6.15) follows now from

$$
Q_{d}(z)=\lim _{x \rightarrow \infty} \frac{w_{11}(x, z)}{w_{21}(x, z)}=\lim _{x \rightarrow \infty} z \frac{s_{2}\left(x, z^{2}\right)}{c_{2}\left(x, z^{2}\right)}=z q\left(z^{2}\right) .
$$

Funding Open Access funding enabled and organized by Projekt DEAL.

Data Availibility Data sharing not applicable to this article as no datasets were generated or analysed during the current study.

Open Access This article is licensed under a Creative Commons Attribution 4.0 International License, which permits use, sharing, adaptation, distribution and reproduction in any medium or format, as long as you give appropriate credit to the original author(s) and the source, provide a link to the Creative Commons licence, and indicate if changes were made. The images or other third party material in this article are included in the article's Creative Commons licence, unless indicated otherwise in a credit line to the material. If material is not included in the article's Creative Commons licence and your intended use is not permitted by statutory regulation or exceeds the permitted use, you will need to obtain permission directly from the copyright holder. To view a copy of this licence, visit http://creativecommons.org/licenses/by/4.0/.

\section{References}

1. Arens, R.: Operational calculus of linear relations. Pac. J. Math. 11, 9-23 (1961)

2. Arov, D.Z., Dym, H.: Bitangential Direct and Inverse Problems for Systems of Integral and Differential Equations. Cambridge Univ. Press, Cambridge (2012)

3. Atkinson, F.: Discrete and Continuous Boundary Problems, Mathematics in Science and Engineering, vol. 8. Academic Press, New York (1964)

4. Bennewitz, C.: Symmetric relations on a Hilbert space. Lect. Notes Math. 280, 212-218 (1972)

5. Bennewitz, C.: Spectral asymptotics for Sturm-Liouville equations. Proc. Lond. Math. Soc. 59, 294 338 (1989)

6. Berezansky, Y.M., Sheftel, Z.G., Us, G.F.: Functional Analysis, vol. I. Operator Theory: Advances and Applications, vol. 85. Birkhäuser, Basel (1996)

7. de Branges, L.: Some Hilbert spaces of entire functions II. Trans. Am. Math. Soc. 99, 118-152 (1961) 
8. de Branges, L.: Some Hilbert spaces of entire functions IV. Trans. Am. Math. Soc. 105, 43-83 (1962)

9. de Branges, L.: Hilbert Spaces of Entire Functions. Prentice Hall, Englewood Cliffs (1968)

10. Derkach, V.A., Malamud, M.M.: Generalized resolvents and the boundary value problems for Hermitian operators with gaps. J. Funct. Anal. 95, 1-95 (1991)

11. Derkach, V.A., Malamud, M.M.: The extension theory of Hermitian operators and the moment problem. J. Math. Sci. 73, 141-242 (1995)

12. Eckhardt, J., Teschl, G.: Sturm-Liouville operators with measure-valued coefficients. J. Anal. Math. 120, 151-224 (2013)

13. Eckhardt, J., Gesztesy, F., Nichols, R., Teschl, G.: Weyl-Titchmarsh theory for Sturm-Liouville operators with distributional potentials. Opusc. Math. 33, 467-563 (2013)

14. Everitt, W.N.: On the limit point classification of second-order differential operators. J. Lond. Math. Soc. 41, 531-544 (1966)

15. Everitt, W.N., Giertz, M.: A Dirichlet type result for ordinary differential operators. Math. Ann. 203, 119-128 (1973)

16. Everitt, W.N.: A note on the Dirichlet condition for second-order differential expressions. Can. J. Math. 28, 312-320 (1976)

17. Evans, W.D., Everitt, W.N.: A return to the Hardy-Littlewood integral inequality. Proc. R. Soc. Lond. Ser A 380, 447-486 (1982)

18. Feller, W.: Generalized second order differential operators and their lateral conditions. Ill. J. Math. 1, 459-504 (1957)

19. Feller, W.: The birth and death processes as diffusion processes. J. Math. Pures Appl. 38, 301-345 (1959)

20. Ghatasheh, A., Weikard, R.: Spectral theory for systems of ordinary differential equations with distributional coefficients. J. Differ. Equ. 268(6), 2752-2801 (2020)

21. Gorbachuk, V.I., Gorbachuk, M.L.: Boundary Value Problems for Operator Differential Equations. Kluwer Academic Publishers Group, New York (1991)

22. Hewitt, E.: Integration by Parts for Stieltjes Integrals. Am. Math. Mon. 67(5), 419-423 (1960)

23. Ito, K., McKean, H.P.: Diffusion Processes and Their Sample Paths. Springer, Berlin (1965)

24. Kac, I.S.: Linear relations generated by a canonical differential equation of phase dimension 2 and decomposability in eigenfunctions. Algebra i Analiz 14(3), 86-120 (2002)

25. Kac, I.S., Kreĭn, M.G.: Criteria for the discreteness of the spectrum of a singular string, (Russian) Izv. Vysš. Učebn. Zaved. Matematika 2, 136-153 (1958)

26. Kac, I.S., Krĕn, M.G.: $R$-functions-analytic functions mapping the upper halfplane into itself, Supplement I to the Russian edition of F.V. Atkinson, Discrete and continuous boundary problems, Mir, Moscow, 1968 (Russian). English translation: Amer. Math. Soc. Transl. Ser. (2) 103 (1974), 1-18

27. Kac, I.S., Krein, M.G.: On the spectral functions of the string, Supplement II to the Russian edition of F. V. Atkinson, Discrete and continuous boundary problems, Mir, Moscow: Russian. English translation: Amer. Math. Soc. Transl. (2) 103(1974), 19-102 (1968)

28. Kalf, H.: Remarks on some Dirichlet type results for semibounded Sturm-Liouville operators. Math. Ann. 210, 197-205 (1974)

29. Kaltenback, H., Winker, H., Woracek, H.: Symmetric relations of finite negativity. In: Förster, K.H., Jonas, P., Langer, H. (eds.) Operator Theory in Krein Spaces and Nonlinear Eigenvalue Problems. Operator Theory: Advances and Applications, vol. 162, pp. 191-210. Basel, Birkhäuser (2006)

30. Kaltenbäck, M., Winkler, H., Woracek, H.: Strings, dual strings, and related canonical systems. Math. Nachr. 280(13-14), 1518-1536 (2007)

31. Kasahara, Y.: Spectral theory of generalized second order differential operators and its applications to Markov processes. Jpn. J. Math. (N.S.) 1(1), 67-84 (1975/1976)

32. Kochubei, A.N.: On extentions of symmetric operators and symmetric binary relations. Matem. Zametki 17(1), 41-48 (1975)

33. Kostenko, A.: The similarity problem for indefinite Sturm-Liouville operators and the HELP inequality. Adv. Math. 246, 368-413 (2013)

34. Krein, M.G., Langer, H.: Continuation of Hermitian positive definite functions and related questions. Integr. Equ. Oper. Theory 78, 1-69 (2014)

35. Langer, H., Schenk, W.: Generalized second-order differential operators, corresponding gap diffusions and superharmonic transformations. Math. Nachr. 148, 746 (1990)

36. Lesch, M., Malamud, M.: On the deficiency indices and self-adjointness of symmetric Hamiltonian systems. J. Differ. Equ. 189(2), 556-615 (2003) 
37. Malamud, M.: On the formula of generalized resolvents of a nondensely defined Hermitian operator. Ukr. Mat. Zh. 44(12), 1658-1688 (1992)

38. Mandl, P.: Analytical Treatment of One-Dimensional Markov Processes. Springer, Berlin (1968)

39. Mogilevskii, V.: Spectral and pseudospectral functions of Hamiltonian systems: development of the results by Arov-Dym and Sakhnovich. Methods Funct. Anal. Topol. 21(4), 370-402 (2015)

40. Strelnikov, D.: Boundary triples for integral systems on finite intervals. J. Math. Sci. 231(1), 83-100 (2018)

41. Strelnikov, D.: Boundary triples for integral systems on the half-line. Methods Funct. Anal. Topol. 25(3), 84-96 (2019)

42. Winkler, H.: The inverse spectral problem for canonical systems. Integral Equ. Oper. Theory 22, 360374 (1995)

Publisher's Note Springer Nature remains neutral with regard to jurisdictional claims in published maps and institutional affiliations. 